TRANSACTIONS OF THE

AMERICAN MATHEMATICAL SOCIETY

Volume 351, Number 5, Pages 1989-2023

S 0002-9947(99)02084-X

Article electronically published on January 26, 1999

\title{
ON THE CONTRIBUTION OF THE COULOMB SINGULARITY OF ARBITRARY CHARGE TO THE DIRAC HAMILTONIAN
}

\author{
JINGBO XIA
}

\begin{abstract}
We study the self-adjoint extensions of the Dirac operator $\alpha \cdot(p-$ $B)+\mu_{0} \beta-W$, where the electrical potential $W$ contains a Coulomb singularity of arbitrary charge and the magnetic potential $B$ is allowed to be unbounded at infinity. We show that if the Coulomb singularity has the form $v(r) / r$ where $v$ has a limit at 0 , then, for any self-adjoint extension of the Dirac operator, removing the singularity results in a Hilbert-Schmidt perturbation of its resolvent.
\end{abstract}

\section{INTRODUCTION}

In this paper we investigate the deficiency indices and the resolvent perturbation of the Dirac operator

$$
\alpha \cdot(p-B)+\mu_{0} \beta-W,
$$

where $\mu_{0} \in \mathbf{R}$ is the rest energy of the particle in question and $(B, W)=\left(B_{1}, B_{2}\right.$, $\left.B_{3}, W\right)$ represents an electromagnetic field. By deficiency indices we mean, of course, those of the minimal symmetric operator on the domain $C_{c}^{\infty}\left(\mathbf{R}^{3}\right) \otimes \mathbf{C}^{4}$. In a typical atomic model, one decomposes the electrical part of the potential as

$$
W=V+V_{1}+V_{2},
$$

where $V$ accounts for the Coulomb singularity if the nucleus is regarded as a point charge, $V_{1}$ is bounded on $\mathbf{R}^{3}$, and $V_{2}$ represents an external electrical field, which is bounded on every bounded subset of $\mathbf{R}^{3}$ and which can be unbounded at infinity. The magnetic potential $B$ is also assumed to be locally bounded.

We will further assume that $V$ is a radial function and has the form

$$
V(r)=v(r) / r,
$$

where $v$ is a real-valued, bounded, measurable function with a bounded support. ( $V_{1}$ accounts for the long-range part of the Coulomb potential and other correction terms.) Moreover, we assume that

$$
v\left(0^{+}\right)=\lim _{\epsilon \downarrow 0} v(\epsilon)
$$

exists. If the Coulomb field is produced by a nucleus of atomic number $Z$, one naturally takes $v\left(0^{+}\right)$to be $Z e^{2} / \hbar c \approx Z / 137.036$. In such an atomic model, the

Received by the editors January 15, 1996 and, in revised form, February 27, 1997.

1991 Mathematics Subject Classification. Primary 47A10, 47F05, 81Q10, 81V45.

Key words and phrases. Dirac operator, spectrum, perturbation.

Research supported in part by National Science Foundation grant DMS-9400600.

(C)1999 American Mathematical Society 
deviation of $v(r) / r$ from the pure Coulomb potential $\left(Z e^{2} / \hbar c\right) / r$ arises from various screening effects in atoms [9].

To explain what we will do, let us first recall what is known ${ }^{1}$. In [8], [11], [13], Dirac operators of the form $\alpha \cdot p+\mu_{0} \beta-U$ was studied. In these papers, $U=U_{1}+U_{2}$, where $U_{1}$ accounts for the Coulomb singularity at 0 and $U_{2}$ is bounded. These authors showed that if $\left|U_{1}(x)\right| \leq \nu /|x|$ with some $0 \leq \nu<1$ (which covers atomic numbers up to $Z=137$ ), then self-adjoint extensions of $\alpha \cdot p+\mu_{0} \beta-U$ can be naturally constructed. More precisely, for such a $U_{1}$ there is a unique selfadjoint extension of the minimal operator which has the property that its domain is contained in that of $\left|\alpha \cdot p+\mu_{0} \beta\right|^{1 / 2}$ [8]. If, furthermore, $U_{2}$ vanishes at infinity, then the essential spectrum of this particular self-adjoint extension is contained in that of the free Dirac operator $\alpha \cdot p+\mu_{0} \beta$ [8]. Also see [5].

In these investigations the assumption that $\left|U_{1}(x)\right| \leq \nu /|x|$ with some $0 \leq \nu<1$ seems to be crucial, and the techniques employed there seem to break down drastically once this condition is dropped. On the other hand, one's natural curiosity does not stop at $Z=137$. Whatever the physical reality regarding nuclei with large charges may be, mathematically one is entitled to ask what happens to the selfadjoint extensions and the essential spectrum of the Dirac operator when $Z>137$ ? And this, as we will see in Section 6, is where interesting analysis gets involved.

Our investigation was originally motivated by this question. However we will go somewhat further. Notice that in the literature cited above, the potential for the Dirac operator is required to be bounded near infinity. In other words, these results do not cover the case where there is a genuine external electromagnetic field, whose potential is generally unbounded at infinity. We will show how to realize the Dirac Hamiltonian as a self-adjoint operator when there are singularities at both 0 and infinity.

Our approach to the problem is as follows. First we switch off the external field $\left(B, V_{2}\right)$ and consider the minimal symmetric operator

$$
\alpha \cdot p+\mu_{0} \beta-V-V_{1}
$$

on the domain $C_{c}^{\infty}\left(\mathbf{R}^{3}\right) \otimes \mathbf{C}^{4}$, where $V$ and $V_{1}$ are as described in the above. We will show that this operator always has finite, equal deficiency indices and that, if $\left(\max \left\{0, \ell^{2}-(1 / 4)\right\}\right)^{1 / 2}<\left|v\left(0^{+}\right)\right|<\left((\ell+1)^{2}-(1 / 4)\right)^{1 / 2}$ for some $\ell \in \mathbf{Z}_{+}$, then these deficiency indices are $(2 \ell(\ell+1), 2 \ell(\ell+1))$. If $v=v\left(0^{+}\right)$in a neighborhood of 0 , then, of course, this result can be easily deduced by solving elementary differential equations. But the non-constancy of $v$ brings substantial complications to the proof. Thus the screening effects represented by the non-constancy of $v$ have no bearing on the deficiency indices of the Dirac operator except, possibly, when $\left|v\left(0^{+}\right)\right|$equals one of the critical values $\left\{\left((\ell+1)^{2}-(1 / 4)\right)^{1 / 2}: \ell \in \mathbf{Z}_{+}\right\}$. On the other hand, if $\left|v\left(0^{+}\right)\right|=\left((\ell+1)^{2}-(1 / 4)\right)^{1 / 2}$ for some $\ell \in \mathbf{Z}_{+}$, our result clearly suggests that the deficiency indices of the Dirac operator cannot be determined without further knowledge of the behavior of $v$ near 0 .

We will then show that if $A$ is any self-adjoint extension of the minimal operator $\alpha \cdot p+\mu_{0} \beta-V-V_{1}$ and if $A_{0}$ is the closure of $\alpha \cdot p+\mu_{0} \beta-V_{1}$, which is necessarily self-adjoint since $V_{1}$ is assumed to be bounded, then

$$
(A-z)^{-1}-\left(A_{0}-z\right)^{-1}
$$

\footnotetext{
${ }^{1}$ For those who are not familiar with the subject, Section V.5.4 of [6] provides a brief summary of background information.
} 
is a Hilbert-Schmidt operator whenever $z \in \mathbf{C} \backslash \mathbf{R}$. We emphasize that this result is valid for every $v\left(0^{+}\right) \in \mathbf{R}$. This result in particular implies that $\sigma_{\text {ess }}(A)$ is determined by $V_{1}$ only.

Next we switch on the the external field. We may, of course, represent the external field collectively by a single matrix-valued potential $\mathcal{E}$, which is assumed to be bounded on every bounded subset of $\mathbf{R}^{3}$. For any self-adjoint extension $A$ of $\alpha \cdot p+\mu_{0} \beta-V-V_{1}$, let

$$
\mathcal{D}_{c}(A)=\{\varphi \in \text { domain of } A: \varphi \text { has a bounded support }\} .
$$

We will show that $A-\mathcal{E}$ is essentially self-adjoint on $\mathcal{D}_{c}(A)$. For the closure $A_{0}$ of $\alpha \cdot p+\mu_{0} \beta-V_{1}, A_{0}-\mathcal{E}$ is also essentially self-adjoint on $\mathcal{D}_{c}(A)$. We will prove that

$$
(A-\mathcal{E}-z)^{-1}-\left(A_{0}-\mathcal{E}-z\right)^{-1}
$$

is a Hilbert-Schmidt operator whenever $z \in \mathbf{C} \backslash \mathbf{R}$. These results will be deduced from the results stated in the two preceding paragraphs. To make this possible we must fully exploit the fact that $\alpha \cdot p$ is a first order partial differential operator.

In contrast to [8], [11], [13], we rely on the radial-spherical decomposition of the free Dirac operator $\alpha \cdot p$, which produces a bunch of ordinary differential operators. We will deal with the resolvent perturbation and the deficiency indices of these operators in Sections 1 and 2. After this is taken care of, we prove the above stated results in Sections 3-5.

Section 6 deals with the eigenvectors of the self-adjoint extensions of $\alpha \cdot p+\mu_{0} \beta-$ $V-V_{1}$. We will show, under very reasonable restrictions on $v$, that if $\left|v\left(0^{+}\right)\right|>1$ and if $V_{1}$ has the right long-range behavior, then every self-adjoint extension $A$ of the minimal operator has the following property: There exist an infinite number of eigenstates $\varphi$ corresponding to eigenvalues of $A$ in $\left(-\left|\mu_{0}\right|,\left|\mu_{0}\right|\right)$ such that the generalized expectation values $\left\langle V+V_{1}\right\rangle_{\varphi}$ and $\langle\alpha \cdot p\rangle_{\varphi}$ of the potential energy and the kinetic energy (in units $\hbar=c=1$ ) are infinite. In particular every $s$-state exhibits this behavior. This phenomenon seems to be a manifestation of what must be the relativistically correct version of the uncertainty principle.

Throughout the paper, identity operators will be routinely suppressed to simplify notation. Thus, for example, in (6.5), the first $d^{2} / d t^{2}$ means $\left(d^{2} / d t^{2}\right) \otimes I_{4}$ while the second and the third mean $\left(d^{2} / d t^{2}\right) \otimes I_{2}$ ( $I_{n}$ being the $n \times n$ identity matrix).

\section{The Resolvents of the RAdial operators}

Throughout this section,

$$
V(t)=v(t) / t
$$

on $(0, \infty)$, where $v(t)$ is a bounded, real-valued, measurable function with the following properties:

(i) There is a positive number $d$ such that $v=0$ on $(d, \infty)$.

(ii) The limit $v\left(0^{+}\right)=\lim _{\epsilon \downarrow 0} v(\epsilon)$ exists.

For each $\ell \in \mathbf{Z}_{+}$, define the differential operator

$$
D_{\ell}=\left[\begin{array}{cc}
(1+\ell) / t & d / d t \\
-d / d t & -(1+\ell) / t
\end{array}\right]
$$

on the domain $C_{c}^{\infty}(0, \infty) \otimes \mathbf{C}^{2}$ in the Hilbert space

$$
H=L^{2}(0, \infty) \otimes \mathbf{C}^{2}=L^{2}(0, \infty) \oplus L^{2}(0, \infty) .
$$


It is elementary that the deficiency indices of $D_{\ell}$ in $H$ are $(0,0)$. The easiest way to see this fact is to solve an elementary linear system of differential equations.

Indeed if we define

$$
A_{\ell, n}=\left[\begin{array}{cc}
1+\ell & n \\
-n & -(1+\ell)
\end{array}\right]
$$

for each $n \in \mathbf{Z}$, then for any column vector $a \in \mathbf{C}^{2}$, we have $D_{\ell} a t^{n}=A_{\ell, n} a t^{n-1}$ if $D_{\ell}$ is also regarded as an operator on polynomials in $t$ and $1 / t$. Note that $A_{\ell, n}$ is invertible for $n \neq \pm(1+\ell)$. Let

$$
s_{+}=\left[\begin{array}{l}
1 \\
1
\end{array}\right] \text { and } s_{-}=\left[\begin{array}{c}
1 \\
-1
\end{array}\right] \text {. }
$$

Then

$$
A_{\ell, n}^{-1} s_{+}=\frac{-(n+\ell+1)}{n^{2}-(\ell+1)^{2}} s_{-} \text {and } A_{\ell, n}^{-1} s_{-}=\frac{(n-\ell-1)}{n^{2}-(\ell+1)^{2}} s_{+} .
$$

In particular, there is a $c_{\ell} \in \mathbf{R}$ such that

$$
A_{\ell, \ell}^{-1} A_{\ell, \ell-1}^{-1} \ldots A_{\ell,-\ell+1}^{-1} A_{\ell,-\ell}^{-1} s_{+}=c_{\ell} s_{-} .
$$

Therefore

$$
\frac{c_{\ell}}{2 \ell+2} A_{\ell, \ell+1} s_{+}=A_{\ell, \ell}^{-1} A_{\ell, \ell-1}^{-1} \ldots A_{\ell,-\ell+1}^{-1} A_{\ell,-\ell}^{-1} s_{+} .
$$

From this identity it follows that for any $z \in \mathbf{C}$, the $2 \times 2$ linear system of differential equations $D_{\ell} u=z u$ has the solution

$$
\begin{aligned}
u_{1}= & t^{-\ell-1}\left(s_{+}+\sum_{n=1}^{2 \ell+1} z^{n} t^{n} A_{\ell,-\ell-1+n}^{-1} \ldots A_{\ell,-\ell}^{-1} s_{+}\right) \\
& +\frac{c_{\ell} z^{2 \ell+2} t^{\ell+1}}{2 \ell+2}\left(s_{+}+\sum_{n=1}^{\infty} z^{n} A_{\ell, \ell+1+n}^{-1} \ldots A_{\ell, \ell+1+1}^{-1} t^{n} s_{+}\right) .
\end{aligned}
$$

This system also has the solution

$$
u_{2}=t^{\ell+1}\left(s_{-}+\sum_{n=1}^{\infty} z^{n} t^{n} A_{\ell, \ell+1+n}^{-1} \ldots A_{\ell, \ell+1+1}^{-1} s_{-}\right) .
$$

It is obvious that these power series in $t$ converge uniformly on any bounded interval. Thus, in the case $z \in \mathbf{C} \backslash \mathbf{R}$, if $\tilde{u}$ also solves $D_{\ell} u=z u$ and has the additional property that $\tilde{u}$ and $D_{\ell} \tilde{u}$ both belong to $H$, then $\tilde{u}$ must be a scalar multiple of $u_{2}$. Using the property $\left|u_{2}(t)\right| \leq M t^{\ell+1}$ for $t$ near 0 and a routine approximation argument, we see that $\tilde{u}$ belongs to the domain for the closure of the minimal operator $D_{\ell}$ on $C_{c}^{\infty}(0, \infty) \otimes \mathbf{C}^{2}$. Since $D_{\ell}$ is symmetric, this is possible only if $\tilde{u}=0$. Hence the deficiency indices of $D_{\ell}$ are $(0,0)$. In other words, every $D_{\ell}$ is essentially self-adjoint on $C_{c}^{\infty}(0, \infty) \otimes \mathbf{C}^{2}$.

Define

$$
D_{00}=\left[\begin{array}{cc}
0 & d / d t \\
-d / d t & 0
\end{array}\right] .
$$

Then, with the initial domain $C_{c}^{\infty}(0, \infty) \otimes \mathbf{C}^{2}$, this operator has deficiency indices $(1,1)$ in $L^{2}(0, \infty) \otimes \mathbf{C}^{2}$. The same, of course, is true if $(0, \infty)$ is replaced by $(a, \infty)$ for any positive $a$. Suppose that $Q$ is a real-valued measurable function on $(0, \infty)$ which is locally integrable. Then $D_{\ell}-Q$ has deficiency indices $(n, n)$ because the 
coefficients of this differential operator are real. Now if $Q$ is bounded on $(a, \infty)$ for every $a>0$, then $n \leq 1$. This is because on $(a, \infty), D_{\ell}-Q$ differs from $D_{00}$ by a bounded term. Thus, with the domain $C_{c}^{\infty}(a, \infty) \otimes \mathbf{C}^{2}, D_{\ell}-Q$ has deficiency indices $(1,1)$ in $L^{2}(a, \infty) \otimes \mathbf{C}^{2}$. That is, for any $z \in \mathbf{C} \backslash \mathbf{R}$, the differential equation $\left(D_{\ell}-Q\right) u=z u$ has, up to scalar multiples, exactly one non-trivial solution in $L^{2}(a, \infty) \otimes \mathbf{C}^{2}$. Hence $n \leq 1$.

We will refer to the operators $D_{\ell}$ and $D_{\ell}-V$ on $C_{c}^{\infty}(0, \infty) \otimes \mathbf{C}^{2}$ as minimal operators. We will denote the closure of $D_{\ell}$, which is a self-adjoint operator, by the same symbol. Similarly, any unspecified self-adjoint extension of the minimal operator $D_{\ell}-V$ will also be denoted by the same symbol.

Recall that a collection $\mathcal{C}$ of bounded operators on a Hilbert space $\mathcal{X}$ is said to be a norm ideal [3] if it is an ideal and is equipped with a norm $\|.\|_{\mathcal{C}}$ satisfying the following conditions: $(1)\left(\mathcal{C},\|.\|_{\mathcal{C}}\right)$ is a Banach space. (2) For any $T \in \mathcal{C}$ and any bounded operators $F, G$ on $\mathcal{X},\|F T G\|_{\mathcal{C}} \leq\|F\|\|T\|_{\mathcal{C}}\|G\|$. (3) If $T \in \mathcal{C}$, then $T^{*} \in \mathcal{C}$ and $\|T\|_{\mathcal{C}}=\left\|T^{*}\right\|_{\mathcal{C}}$. (4) $\|T\| \leq\|T\|_{\mathcal{C}}$ for every $T \in \mathcal{C}$, and the equality holds whenever $\operatorname{rank}(T)=1$.

Lemma 1.1. Suppose that $A$ and $B$ are (not necessarily bounded) self-adjoint operators on a Hilbert space $\mathcal{X}$. Suppose that $\mathcal{C}$ is a norm ideal consisting of compact operators on $\mathcal{X}$.

(a) Suppose that $\left(A-z_{0}\right)^{-1}-\left(B-z_{0}\right)^{-1} \in \mathcal{C}$ for some $z_{0} \in \mathbf{C} \backslash \mathbf{R}$. Then for any bounded self-adjoint operator $C$ on $\mathcal{X}$ and any $z \in \mathbf{C} \backslash \mathbf{R}$, we have $(A+C-z)^{-1}-$ $(B+C-z)^{-1} \in \mathcal{C}$.

(b) Suppose that $S$ is a bounded operator on $\mathcal{X}$ and $z_{0} \in \mathbf{C} \backslash \mathbf{R}$ such that $S\left(A-z_{0}\right)^{-1} \in \mathcal{C}$. Then $S(A+C-z)^{-1} \in \mathcal{C}$ for every bounded self-adjoint operator $C$ on $\mathcal{X}$ and for every $z$ in the open half-plane that contains $z_{0}$.

The proof of this lemma is completely elementary and will be omitted.

Lemma 1.2. Let $E$ be a bounded Borel set in $(0, \infty)$. Then for any $z \in \mathbf{C} \backslash \mathbf{R}$, $\chi_{E}\left(D_{\ell}-z\right)^{-1}$ is a Hilbert-Schmidt operator.

Proof. Let us first show that if $D$ is any self-adjoint extension of $D_{00}$ with the initial domain $C_{c}^{\infty}(0, \infty) \otimes \mathbf{C}^{2}$, then for any $\lambda \in \mathbf{R} \backslash\{0\}, \chi_{E}(D-\lambda i)^{-1}$ is a HilbertSchmidt operator. Equivalently, we want to show that $\chi_{E}(D-\lambda i)^{-1}(D+\lambda i)^{-1} \chi_{E}=$ $\chi_{E}\left(D^{2}+\lambda^{2}\right)^{-1} \chi_{E}$ is a trace class operator.

Suppose that $E \subset(0, R)$. Let $A$ be the self-adjoint extension in $L^{2}(0, R) \otimes \mathbf{C}^{2}$ of the operator $-d^{2} / d t^{2}$ on $C_{c}^{\infty}(0, R) \otimes \mathbf{C}^{2}$ such that $\left\{e^{2 k \pi i t / R} v: k \in \mathbf{Z}, v \in \mathbf{C}^{2}\right\}$ are its eigenvectors. Then $\left(A+\lambda^{2}\right)^{-1}$ is a trace class operator on $L^{2}(0, R) \otimes \mathbf{C}^{2}$. Since $D^{2}$ is a self-adjoint extension of $-d^{2} / d t^{2}$ in $L^{2}(0, \infty) \otimes \mathbf{C}^{2}$, if we consider both $\left(D^{2}+\lambda^{2}\right)^{-1} \chi_{(0, R)}$ and $\left(A+\lambda^{2}\right)^{-1}$ as operators from $L^{2}(0, R) \otimes \mathbf{C}^{2}$ to $L^{2}(0, \infty) \otimes \mathbf{C}^{2}$ in the natural way, then they agree on the closure of $\left(\lambda^{2}-d^{2} / d t^{2}\right) C_{c}^{\infty}(0, R) \otimes \mathbf{C}^{2}$. Thus, as such operators, $\left(D^{2}+\lambda^{2}\right)^{-1} \chi_{(0, R)}$ and $\left(A+\lambda^{2}\right)^{-1}$ differ by an operator of rank at most 4 . This proves that $\left(D^{2}+\lambda^{2}\right)^{-1} \chi_{(0, R)}$ is a trace class operator and so is $\chi_{E}\left(D^{2}+\lambda^{2}\right)^{-1} \chi_{E}$.

For any $\varphi \in C_{c}^{\infty}(0, \infty) \otimes \mathbf{C}^{2}$, we have

$$
\left\|\left(D_{00}-\lambda i\right) \varphi\right\|^{2}=\left\langle\left(D_{00}^{2}+\lambda^{2}\right) \varphi, \varphi\right\rangle \leq\left\langle\left(D_{\ell}^{2}+\lambda^{2}\right) \varphi, \varphi\right\rangle=\left\|\left(D_{\ell}-\lambda i\right) \varphi\right\|^{2} .
$$

This implies that there exists a contraction $T$ on $H$ such that $D_{00}-\lambda i=$ $T\left(D_{\ell}-\lambda i\right)$ on $C_{c}^{\infty}(0, \infty) \otimes \mathbf{C}^{2}$. Thus

$$
\left(D_{\ell}-\lambda i\right)^{-1}\left(D_{\ell}-\lambda i\right) \varphi=(D-\lambda i)^{-1}\left(D_{00}-\lambda i\right) \varphi=(D-\lambda i)^{-1} T\left(D_{\ell}-\lambda i\right) \varphi
$$


whenever $\varphi \in C_{c}^{\infty}(0, \infty) \otimes \mathbf{C}^{2}$. Since $\left(D_{\ell}-\lambda i\right) C_{c}^{\infty}(0, \infty) \otimes \mathbf{C}^{2}$ is dense in $H$, we have

$$
\chi_{E}\left(D_{\ell}-\lambda i\right)^{-1}=\chi_{E}(D-\lambda i)^{-1} T .
$$

The lemma follows from this and Lemma 1.1 .

For each $b \in \mathbf{R}$, let $V_{b}(t)=b \chi_{(0, d)}(t) / t$. Let $\Phi_{b, \ell}$ denote fundamental solution matrix of the $2 \times 2$ linear system of differential equations $\left(D_{\ell}-V_{b}\right) u=0$. If $(1+\ell)^{2}-b^{2} \neq 0$, then on the interval $(0, d)$,

$$
\Phi_{b, \ell}(t)=\left[\begin{array}{cc}
\sqrt{(1+\ell)^{2}-b^{2}} t \sqrt{(1+\ell)^{2}-b^{2}} & \sqrt{(1+\ell)^{2}-b^{2}} t^{-\sqrt{(1+\ell)^{2}-b^{2}}} \\
-(1+\ell-b) t \sqrt{(1+\ell)^{2}-b^{2}} & (1+\ell-b) t^{-\sqrt{(1+\ell)^{2}-b^{2}}}
\end{array}\right] .
$$

In the cases $1+\ell-b=0$ and $1+\ell+b=0$, we have

$$
\Phi_{b, \ell}(t)=\left[\begin{array}{cc}
1 & -(1+\ell+b) \log t \\
0 & 1
\end{array}\right] \text { and }\left[\begin{array}{cc}
1 & 0 \\
-(1+\ell-b) \log t & 1
\end{array}\right]
$$

respectively on $(0, d)$.

Let us now consider the $2 \times 2$ linear system of differential equations

$$
\left(D_{\ell}-V_{b}-z\right) u=0
$$

for arbitrary $z \in \mathbf{C}$. To solve this equation, one sets $u=\Phi_{b, \ell} c$ and finds $B \Phi_{b, \ell} c^{\prime}=$ $z \Phi_{b, \ell} c$ or, equivalently, $c^{\prime}=z \Phi_{b, \ell}^{-1} B^{-1} \Phi_{b, \ell} c$. Here

$$
B=\left[\begin{array}{cc}
0 & 1 \\
-1 & 0
\end{array}\right]
$$

Let $A_{b, \ell}(t)=\Phi_{b, \ell}^{-1}(t) B^{-1} \Phi_{b, \ell}(t)$. When $(1+\ell)^{2}-b^{2}<1 / 4, A_{b, \ell}$ is integrable near 0 .

Lemma 1.3. Let $\Psi_{b, \ell, z}$ denote the fundamental solution matrix for the linear system (1.2).

(a) If $0<(1+\ell)^{2}-b^{2}<1 / 4$, then $\Psi_{b, \ell, z}(t)=\Phi_{b, \ell}(t)(1+G(t))$, where

$$
G=\left[\begin{array}{ll}
g_{11} & g_{12} \\
g_{21} & g_{22}
\end{array}\right]
$$

with $\left|g_{i j}(t)\right| \leq O(t)$ for $(i, j) \neq(1,2)$ and $g_{12}(t)=O\left(t^{1-2 \sqrt{(1+\ell)^{2}-b^{2}}}\right)$ when $t$ is near 0 .

(b) If $(1+\ell)^{2}-b^{2}=0$, then $\Psi_{b, \ell, z}(t)=\Phi_{b, \ell}(t)\left(1+O\left(t(\log t)^{2}\right)\right)$ for $t$ near 0 .

(c) If $(1+\ell)^{2}-b^{2}<0$, then $\Psi_{b, \ell, z}(t)=\Phi_{b, \ell}(t)(1+O(t))$ for $t$ near 0 .

Proof. By the paragraph preceding the lemma, we have

where

$$
\Psi_{b, \ell, z}(t)=\Phi_{b, \ell}(t)\left[1+\int_{0}^{t} A_{b, \ell}(s) K(s) d s\right]
$$

$$
K(s)=z+\sum_{n=1}^{\infty} z^{n+1} \int_{0}^{s} A_{b, \ell}\left(s_{n}\right) \int_{0}^{s_{n}} A_{b, \ell}\left(s_{n-1}\right) \cdots \int_{0}^{s_{2}} A_{b, \ell}\left(s_{1}\right) d s_{1} \ldots d s_{n-1} d s_{n} .
$$

It follows from the integrability of $A_{b, \ell}$ that this series converges uniformly and is bounded near 0 . In the cases (b) and (c), $\left|A_{b, \ell}(s)\right|$ is dominated by $O\left((\log s)^{2}\right)$ and $O(1)$ respectively. 
For (a), let us write $\mu=\sqrt{(1+\ell)^{2}-b^{2}}$ and $\nu=1+\ell-b$. Then

$$
A_{b, \ell}(s)=\frac{1}{2 \mu \nu}\left[\begin{array}{cc}
\nu^{2}-\mu^{2} & -\left(\nu^{2}+\mu^{2}\right) s^{-2 \mu} \\
\left(\nu^{2}+\mu^{2}\right) s^{2 \mu} & \mu^{2}-\nu^{2}
\end{array}\right] .
$$

Since $1-2 \mu>0$,

$$
\left|\int_{0}^{t} A_{b, \ell}(s) \int_{0}^{s} A_{b, \ell}(r) d r d s\right| \leq O(t) .
$$

The desired estimate follows from these relations.

For each $\mu>-1 / 2$, define

$$
\left(T_{\mu} f\right)(t)=t^{-\mu-1} \int_{0}^{t} s^{\mu} f(s) d s .
$$

It follows from a well-known inequality of Hardy that $T_{\mu}$ is a bounded operator on $L^{2}(0, \infty)$. See, for example, [1, Lemma 3.3.9]. We also define

$$
\left(T_{0}^{\prime} f\right)(t)=t^{-1} \int_{0}^{t} \log \left(\frac{t}{s}\right) f(s) d s .
$$

It is easy to see that $T_{0}^{\prime}$ is also a bounded operator on $L^{2}(0, \infty)$. Indeed, by the Cauchy-Schwarz inequality,

$$
\left|\left(T_{0}^{\prime} f\right)(t)\right|^{2} \leq t^{-1} \int_{0}^{t}\left[1+\log \left(\frac{t}{s}\right)\right]^{4} d s \cdot t^{-1} \int_{0}^{t} \frac{|f(s)|^{2}}{\left(1+\log \left(\frac{t}{s}\right)\right)^{2}} d s .
$$

If we set $C_{1}=\int_{0}^{1}(1-\log u)^{4} d u$ and $C_{2}=\int_{1}^{\infty} u^{-1}(1+\log u)^{-2} d u$, then

$$
\begin{aligned}
\int_{0}^{\infty} & \left|\left(T_{0}^{\prime} f\right)(t)\right|^{2} d t \leq C_{1} \int_{0}^{\infty} t^{-1} \int_{0}^{t} \frac{|f(s)|^{2}}{\left(1+\log \left(\frac{t}{s}\right)\right)^{2}} d s d t \\
& =C_{1} \int_{0}^{\infty}|f(s)|^{2}\left[\int_{s}^{\infty} \frac{1}{t\left(1+\log \left(\frac{t}{s}\right)\right)^{2}} d t\right] d s=C_{1} C_{2} \int_{0}^{\infty}|f(s)|^{2} d s .
\end{aligned}
$$

Lemma 1.4. Suppose that $(1+\ell)^{2}-b^{2}<1 / 4$. Define

$$
\left(W_{b, \ell, z} h\right)(t)=t^{-1} \Psi_{b, \ell, z}(t) \int_{0}^{t} \Psi_{b, \ell, z}^{-1}(s) h(s) d s .
$$

Then the operator $\chi_{(0, c)} W_{b, \ell, z} \chi_{(0, c)}$ is bounded for every $0<c<\infty$.

Proof. By Lemma 1.3(c), if $(1+\ell)^{2}-b^{2}<0$, then $\Psi_{b, \ell, z}$ and $\Psi_{b, \ell, z}^{-1}$ are bounded near 0 . Hence in this case the boundedness of $W_{b, \ell, z}$ follows from that of $T_{0}$.

Suppose that $0<(1+\ell)^{2}-b^{2}<1 / 4$. Again, let us write $\mu=\sqrt{(1+\ell)^{2}-b^{2}}$ and $\nu=1+\ell-b$. Let

$$
X=\left[\begin{array}{cc}
\mu & \mu \\
-\nu & \nu
\end{array}\right] \text { and } Y(t)=\left[\begin{array}{cc}
t^{\mu} & 0 \\
0 & t^{-\mu}
\end{array}\right]
$$

Then

$$
\left(W_{b, \ell, z} h\right)(t)=t^{-1} X Y(t)(1+G(t)) \int_{0}^{t}(1+\Gamma(s)) Y^{-1}(s) X^{-1} B^{-1} h(s) d s
$$

where, according to Lemma 1.3(a), the matrices

$$
G(t)=\left[\begin{array}{ll}
g_{11}(t) & g_{12}(t) \\
g_{21}(t) & g_{22}(t)
\end{array}\right] \text { and } \Gamma(s)=\left[\begin{array}{ll}
\gamma_{11}(s) & \gamma_{12}(s) \\
\gamma_{21}(s) & \gamma_{22}(s)
\end{array}\right]
$$


are such that $g_{12}(t)=O\left(t^{1-2 \mu}\right), \gamma_{12}(s)=O\left(s^{1-2 \mu}\right)$, and all other entries are dominated by $O(t)$ or $O(s)$.

Using the boundedness of $T_{\mu}$ and $T_{-\mu}$, we can decompose $W_{b, \ell, z}$ as $W_{b, \ell, z}^{1}+W_{b, \ell, z}^{2}$, where $W_{b, \ell, z}^{2}$ is bounded and

$$
\left(W_{b, \ell, z}^{1} h\right)(t)=t^{-1} X Y_{1}(t)(1+G(t)) \int_{0}^{t}(1+\Gamma(s)) Z_{1}(s) X^{-1} B^{-1} h(s) d s,
$$

with

$$
Y_{1}(t)=\left[\begin{array}{cc}
0 & 0 \\
0 & t^{-\mu}
\end{array}\right] \text { and } Z_{1}(s)=\left[\begin{array}{cc}
s^{-\mu} & 0 \\
0 & 0
\end{array}\right] .
$$

On the other hand, $Y_{1}(t) G(t)=O\left(t^{1-\mu}\right), \Gamma(s) Z_{1}(s)=O\left(s^{1-\mu}\right),-\mu>\mu-1$, and $-1-\mu>\mu-2$. Also

$$
t^{-1} X Y_{1}(t) \int_{0}^{t} Z_{1}(s) X^{-1} B^{-1} h(s) d s=0 .
$$

Therefore the boundedness of $W_{b, \ell, z}^{1}$ follows from that of $T_{-\mu}$ and $T_{1-\mu}$.

Let us consider the case $1+\ell-b=0$; the case $1+\ell+b=0$ can be treated similarly. We have

$$
\left(W_{b, \ell, z} h\right)(t)=t^{-1} \Phi_{b, \ell}(t)(1+G(t)) \int_{0}^{t}(1+\Gamma(s)) \Phi_{b, \ell}^{-1}(s) B^{-1} h(s) d s,
$$

where, by Lemma 1.3(b), $G(t)=O\left(t(\log t)^{2}\right)$ and $\Gamma(s)=O\left(s(\log s)^{2}\right)$ near 0 . Since $\Phi_{b, \ell}(t)=O(-\log t)$ and $\Phi_{b, \ell}^{-1}(s)=O(-\log s), t^{-1} \Phi_{b, \ell}(t) G(t) \chi_{(0, t)}(s) \Phi_{b, \ell}^{-1}(s)$, $t^{-1} \Phi_{b, \ell}(t) \chi_{(0, t)}(s) \Gamma(s) \Phi_{b, \ell}^{-1}(s)$ and $t^{-1} \Phi_{b, \ell}(t) G(t) \chi_{(0, t)}(s) \Gamma(s) \Phi_{b, \ell}^{-1}(s)$ are kernels of bounded operators. Hence it suffices to show that

$$
\left(W_{0} h\right)(t)=t^{-1} \Phi_{b, \ell}(t) \int_{0}^{t} \Phi_{b, \ell}^{-1}(s) B^{-1} h(s) d s
$$

is a bounded operator. Multiplying out the right-hand side in terms of the entries of $\Phi_{b, \ell}$ and $\Phi_{b, \ell}^{-1}$ and the components of $B^{-1} h$, we find that $W_{0}$ is a combination of $T_{0}$ and $T_{0}^{\prime}$. This completes the proof.

Lemma 1.5. Suppose that $\left|v\left(0^{+}\right)\right|>\left[(1+\ell)^{2}-(1 / 4)\right]^{1 / 2}$. Then for any self-adjoint extension of $D_{\ell}-V$, for any bounded Borel set $E \subset(0, \infty)$ and for any $z \in \mathbf{C} \backslash \mathbf{R}$, $\left(D_{\ell}-V-z\right)^{-1} \chi_{E}$ is a Hilbert-Schmidt operator.

Proof. Let $c \in(d, \infty)$ be such that $E \subset(0, c)$. Let us denote $v\left(0^{+}\right)$by $b$. Then $(1+\ell)^{2}-b^{2}<1 / 4$. Define

$$
(R g)(t)=\Psi_{b, \ell, z}(t) \int_{0}^{t} \Psi_{b, \ell, z}^{-1}(s) B^{-1} g(s) d s .
$$

For each $\varphi \in C_{c}^{\infty}(0, \infty) \otimes \mathbf{C}^{2}$, let $f_{\varphi}=\left(D_{\ell}-V_{b}-z\right) \varphi$. It is straightforward to verify that $w_{1}=R f_{\varphi}$ solves the inhomogeneous differential equation $\left(D_{\ell}-V_{b}-z\right) w=f_{\varphi}$. But $w_{2}=\varphi$ is also a solution and has the property that it vanishes near 0 . Because $\varphi$ vanishes in a neighborhood of 0 , so do $f_{\varphi}$ and $w_{1}$. Therefore $w_{1}=w_{2}$. In other words,

$$
R\left(D_{\ell}-V_{b}-z\right) \varphi=\varphi \text { for every } \varphi \in C_{c}^{\infty}(0, \infty) \otimes \mathbf{C}^{2} .
$$

Recall that $V(t)=v(t) / t$. Let $0<\delta<d$ be such that $|b-v(t)|\left\|\chi_{(0, c)} W_{b, \ell, z} \chi_{(0, c)}\right\|$ $\leq 1 / 2$ for $t \in(0, \delta)$. From Lemma 1.4 and this inequality we have that 
$\left(V-V_{b}\right) \chi_{(0, \delta)} R$ is a bounded operator on $L^{2}(0, c) \otimes \mathbf{C}^{2}$ with $\left\|\left(V-V_{b}\right) \chi_{(0, \delta)} R \chi_{(0, c)}\right\| \leq$ $1 / 2$. Thus $\sum_{n=0}^{\infty}\left[\left(V-V_{b}\right) \chi_{(0, \delta)} R\right]^{n}$ is a bounded operator on $L^{2}(0, c) \otimes \mathbf{C}^{2}$. Let

$$
R^{\prime}=R \sum_{n=0}^{\infty}\left[\left(V-V_{b}\right) \chi_{(0, \delta)} R\right]^{n}
$$

From the identity $R\left(D_{\ell}-V_{b}-z\right) \varphi=\varphi$ we deduce

$$
R^{\prime}\left(D_{\ell}-V_{b}-\left(V-V_{b}\right) \chi_{(0, \delta)}-z\right) \varphi=\varphi
$$

for every $\varphi \in C_{c}^{\infty}(0, c) \otimes \mathbf{C}^{2}$.

Let $H^{\prime}$ denote the closure of $\left(D_{\ell}-V_{b}-\left(V-V_{b}\right) \chi_{(0, \delta)}-z\right) C_{c}^{\infty}(0, c) \otimes \mathbf{C}^{2}$ in $L^{2}(0, c) \otimes$ $\mathbf{C}^{2}$ and let $P_{H^{\prime}}$ denote the orthogonal projection onto $H^{\prime}$. The above identity shows that $R^{\prime} P_{H^{\prime}}$ is a bounded operator on $H$ and that $R^{\prime} P_{H^{\prime}}=\chi_{(0, c)} R^{\prime} P_{H^{\prime}}$. Obviously $\chi_{(0, c)} R \chi_{(0, c)}$ is a Hilbert-Schmidt operator. Therefore so is

$$
R^{\prime} P_{H^{\prime}}=\chi_{(0, c)} R \chi_{(0, c)} P_{H^{\prime}}+\chi_{(0, c)} R \chi_{(0, \delta)} \sum_{n=1}^{\infty}\left[\left(V-V_{b}\right) \chi_{(0, \delta)} R\right]^{n} P_{H^{\prime}} .
$$

It is clear if $A$ is any self-adjoint extension of the operator $D_{\ell}-V_{b}-\left(V-V_{b}\right) \chi_{(0, \delta)}$, then $R^{\prime} P_{H^{\prime}}=(A-z)^{-1} P_{H^{\prime}}$. Since the dimension of $\left(L^{2}(0, c) \otimes \mathbf{C}^{2}\right) \ominus H^{\prime}$ is finite, we conclude that $(A-z)^{-1} \chi_{(0, c)}$ is a Hilbert-Schmidt operator.

The function $U=V_{b}+\left(V-V_{b}\right) \chi_{(0, \delta)}-V=\left(V_{b}-V\right) \chi_{[\delta, \infty)}$ is bounded on $(0, \infty)$. Clearly $B=A+U$ is a self-adjoint extension of $D_{\ell}-V$. Therefore

$$
(B-z)^{-1} \chi_{(0, c)}=-(B-z)^{-1} U(A-z)^{-1} \chi_{(0, c)}+(A-z)^{-1} \chi_{(0, c)}
$$

is a Hilbert-Schmidt operator. Finally, if $B^{\prime}$ is another self-adjoint extension of $D_{\ell}-V$, then $(B-z)^{-1}=\left(B^{\prime}-z\right)^{-1}$ on a subspace whose orthgonal complement has dimension at most one. This completes the proof.

Before stating the next proposition, let us recall a well-known fact regarding Hilbert-Schmidt operators. Suppose that $A$ and $B$ are operators on a Hilbert space $\mathcal{X}$ such that $A A^{*}$ and $B^{*} B$ are Hilbert-Schmidt operators and suppose that $T$ is a bounded operator on $\mathcal{X}$. Then $A T B$ is a Hilbert-Schmidt operator on $\mathcal{X}$ with

$$
\|A T B\|_{2} \leq\|T\|\left(\left\|A A^{*}\right\|_{2}\left\|B^{*} B\right\|_{2}\right)^{1 / 2} .
$$

The proof of this inequality is quite simple. Suppose that $\left\{e_{n}\right\}$ and $\left\{f_{n}\right\}$ are orthonormal sets in $\mathcal{X}$ and that $\left\{\lambda_{n}\right\}$ is a sequence of complex numbers where all but a finite number of terms are 0 . Let $K=\sum_{n} \lambda_{n} f_{n} \otimes e_{n}, K_{1}=\sum_{n}\left|\lambda_{n}\right| e_{n} \otimes e_{n}$ and $K_{2}=\sum_{n}\left|\lambda_{n}\right| f_{n} \otimes f_{n}$. Then

$$
\begin{aligned}
|\operatorname{tr}(A T B K)| & =\left|\sum_{n} \lambda_{n}\left\langle A T B e_{n}, f_{n}\right\rangle\right| \leq\|T\| \sum_{n}\left|\lambda_{n}\right|\left\|B e_{n}\right\|\left\|A^{*} f_{n}\right\| \\
& \leq\|T\|\left(\sum_{n}\left|\lambda_{n}\right|\left\langle B^{*} B e_{n}, e_{n}\right\rangle \sum_{n}\left|\lambda_{n}\right|\left\langle A A^{*} f_{n}, f_{n}\right\rangle\right)^{1 / 2} \\
& =\|T\|\left(\operatorname{tr}\left(B^{*} B K_{1}\right) \operatorname{tr}\left(A A^{*} K_{2}\right)\right)^{1 / 2} \\
& \leq\|T\|\left(\left\|B^{*} B\right\|_{2}\left\|A A^{*}\right\|_{2}\left\|K_{1}\right\|_{2}\left\|K_{2}\right\|_{2}\right)^{1 / 2} \\
& =\|T\|\left(\left\|B^{*} B\right\|_{2}\left\|A A^{*}\right\|_{2}\right)^{1 / 2}\|K\|_{2} .
\end{aligned}
$$

This yields the desired estimate for $\|A T B\|_{2}$. 
Proposition 1.6. Let $\ell \in \mathbf{Z}_{+}$and $0<\delta<1$. Suppose that $Q$ is a boundedly supported, real-valued, measurable function satisfying the condition $|Q(t)| \leq$ $(1+\ell-\delta) / t$ on $(0, \infty)$. Then for any self-adjoint extension of the minimal operator $D_{\ell}-Q$ and any $z \in \mathbf{C} \backslash \mathbf{R},\left(D_{\ell}-Q-z\right)^{-1}-\left(D_{\ell}-z\right)^{-1}$ is a Hilbert-Schmidt operator.

Proof. By Lemma 1.1, it suffices to consider $z=-i$. We first observe that

$$
\begin{aligned}
\left(D_{\ell}-i\right) t\left(D_{\ell}+i\right) & =D_{\ell} t D_{\ell}+t+i\left(D_{\ell} t-t D_{\ell}\right)=-\frac{d}{d t} t \frac{d}{d t}+\frac{(1+\ell)^{2}}{t}+t-\sigma_{2} \\
& \geq \frac{1}{t}\left((1+\ell)^{2}-\sigma_{2} t+t^{2}\right) \geq \frac{1}{t}\left((1+\ell)^{2}-t+t^{2}\right),
\end{aligned}
$$

where $\sigma_{2}$ is the second of the usual trio of Pauli spin matrices. Since $(1+\ell)^{2}-t+t^{2}$ is always positive, the above implies

$$
\left(D_{\ell}-i\right) t\left(D_{\ell}+i\right) \geq\left((1+\ell)^{2}-\frac{\delta}{4}\right) \frac{1}{t} \chi_{(0, \delta / 4)} .
$$

Hence

$$
\left\|t^{1 / 2}\left(D_{\ell}+i\right) \varphi\right\| \geq\left((1+\ell)^{2}-\frac{\delta}{4}\right)^{1 / 2}\left\|\chi_{(0, \delta / 4)} t^{-1 / 2} \varphi\right\|
$$

if $\varphi \in C_{c}^{\infty}(0, \infty) \otimes \mathbf{C}^{2}$. Therefore, if $\psi \in\left(D_{\ell}+i\right) C_{c}^{\infty}(0, \infty) \otimes \mathbf{C}^{2}$ and $0<\epsilon<\delta / 4$, then

$$
\left\|t^{1 / 2} \psi\right\| \geq\left((1+\ell)^{2}-\frac{\delta}{4}\right)^{1 / 2}\left\|\chi_{(\epsilon, \delta / 4)} t^{-1 / 2}\left(D_{\ell}+i\right)^{-1} \psi\right\| .
$$

We know that $\left(D_{\ell}+i\right) C_{c}^{\infty}(0, \infty) \otimes \mathbf{C}^{2}$ is dense in $H$.

We claim that (1.4) holds true if we replace $\psi$ with any $g \in H$ which has a bounded support. Let $\zeta$ be a $C^{\infty}$-function on $[0, \infty)$ such that $\zeta=1$ on $[0,1]$ and $\zeta=0$ on $[2, \infty)$. For each $n \in \mathbf{N}$, let $\zeta_{n}(t)=\zeta(t / n)$. Suppose that $\left\{\varphi_{k}\right\}$ is a sequence in $C_{c}^{\infty}(0, \infty) \otimes \mathbf{C}^{2}$ such that $\left\|\left(D_{\ell}+i\right) \varphi_{k}-g\right\| \rightarrow 0$ as $k \rightarrow \infty$. Then

$$
\left(D_{\ell}+i\right) \zeta_{n} \varphi_{k}=\left[D_{\ell}, \zeta_{n}\right] \varphi_{k}+\zeta_{n}\left(D_{\ell}+i\right) \varphi_{k} .
$$

Substituting this into (1.4) and letting $k \rightarrow \infty$, which is permitted since $t^{1 / 2}\left[D_{\ell}, \zeta_{n}\right]$ and $t^{1 / 2} \zeta_{n}$ are bounded, we find that this inequality holds with

$$
\psi=\left[D_{\ell}, \zeta_{n}\right]\left(D_{\ell}+i\right)^{-1} g+\zeta_{n} g=\zeta_{n}^{\prime} B\left(D_{\ell}+i\right)^{-1} g+\zeta_{n} g .
$$

It follows from the definition of $\zeta_{n}$ that $\left\|t^{1 / 2} \zeta_{n}^{\prime}\right\|_{\infty} \leq\left\|\zeta^{\prime}\right\|_{\infty}(2 n)^{1 / 2} / n$. This implies $\lim _{n \rightarrow \infty}\left\|t^{1 / 2} \zeta_{n}^{\prime} B\left(D_{\ell}+i\right)^{-1} g\right\|=0$. Hence (1.4) holds with any boundedly supported $g \in H$ in place of $\psi$. An immediate consequence of this is that

$$
\left\|\chi_{(\epsilon, \delta / 4)} t^{-1 / 2}\left(D_{\ell}+i\right)^{-1} \chi_{(\epsilon, \delta / 4)} t^{-1 / 2}\right\| \leq\left((1+\ell)^{2}-\frac{\delta}{4}\right)^{-1 / 2}<\left(1+\ell-\frac{\delta}{2}\right)^{-1} .
$$

For each $n \geq 2$, define

$$
R_{n, \epsilon}=\sum_{k=0}^{n}\left(D_{\ell}+i\right)^{-1}\left[\chi_{(\epsilon, \delta / 4)} Q\left(D_{\ell}+i\right)^{-1}\right]^{k} .
$$

Let $q=Q \chi_{(0, \delta / 4)}$. Obviously

$$
R_{n, \epsilon}-\left(D_{\ell}+i\right)^{-1}=\left(D_{\ell}+i\right)^{-1} q^{1 / 2} T_{n, \epsilon} q^{1 / 2}\left(D_{\ell}+i\right)^{-1},
$$


where

$$
T_{n, \epsilon}=\chi_{(\epsilon, \delta / 4)}+\sum_{k=2}^{n}\left[\chi_{(\epsilon, \delta / 4)} Q^{1 / 2}\left(D_{\ell}+i\right)^{-1} Q^{1 / 2} \chi_{(\epsilon, \delta / 4)}\right]^{k-1} .
$$

Since $|Q(t)| \leq(1+\ell-\delta) / t$, it follows from (1.5) that there is a $C_{1}>0$ such that

$$
\left\|T_{n, \epsilon}\right\| \leq C_{1}
$$

for all $n \geq 2$ and $0<\epsilon<\delta / 4$.

It follows from the usual uncertainty inequality that if $f \in C_{c}^{\infty}(0, \infty) \otimes \mathbf{C}^{2}$, then

$$
\left\|t^{-1} f\right\|^{2} \leq 4\left\langle D_{\ell}^{2} f, f\right\rangle \leq 4\left\langle\left(D_{\ell}^{2}+1\right) f, f\right\rangle=4\left\|\left(D_{\ell}+i\right) f\right\|^{2} .
$$

This implies that if $g \in\left(D_{\ell}+i\right) C_{c}^{\infty}(0, \infty) \otimes \mathbf{C}^{2}$ and $\kappa>0$, then

$$
\left\|\chi_{(\kappa, \infty)} t^{-1}\left(D_{\ell}+i\right)^{-1} g\right\| \leq 2\|g\| .
$$

Because $\left(D_{\ell}+i\right) C_{c}^{\infty}(0, \infty) \otimes \mathbf{C}^{2}$ is dense in $H$, the above inequality holds for every $g \in H$. Since $\kappa>0$ is arbitrary, this estimate implies that $\left|t^{-1}\left[\left(D_{\ell}+i\right)^{-1} g\right](t)\right|$ is square integrable on $(0, \infty)$ and that its $L^{2}$-norm does not exceed $2\|g\|$. In other words, $t^{-1}\left(D_{\ell}+i\right)^{-1}$ naturally extends to a bounded operator on $H$ and

$$
\lim _{\kappa \rightarrow 0} \chi_{(\kappa, \infty)} t^{-1}\left(D_{\ell}+i\right)^{-1}=t^{-1}\left(D_{\ell}+i\right)^{-1}
$$

in the strong operator topology.

Let $A=\left(D_{\ell}+i\right)^{-1} q^{1 / 2}$ and $B=q^{1 / 2}\left(D_{\ell}+i\right)^{-1}$. Then

$$
A A^{*}=\left(D_{\ell}+i\right)^{-1}|q|\left(D_{\ell}-i\right)^{-1} .
$$

By the preceding paragraph, $|q|\left(D_{\ell}-i\right)^{-1}$ is bounded. Since $q$ has a bounded support, it follows from Lemma 1.2 that $A A^{*}$ is a Hilbert-Schmidt operator. Similarly $B^{*} B$ is also a Hilbert-Schmidt operator. Thus by $(1.7),(1.8)$ and the discussion preceding the proposition, we have

$$
\left\|R_{n, \epsilon}-\left(D_{\ell}+i\right)^{-1}\right\|_{2} \leq C
$$

for $n \geq 3$ and $0<\epsilon<\delta / 4$, where $C=C_{1}\left(\left\|A A^{*}\right\|_{2}\left\|B^{*} B\right\|_{2}\right)^{1 / 2}$.

By (1.6) and (1.10), the strong limit

$$
\lim _{\epsilon \downarrow 0} R_{n, \epsilon}=R_{n}=\sum_{k=0}^{n}\left(D_{\ell}+i\right)^{-1}\left[q(D+i)^{-1}\right]^{k}
$$

exists. Furthermore, (1.11) implies that

$$
\left\|R_{n}-\left(D_{\ell}+i\right)^{-1}\right\|_{2} \leq C .
$$

By (1.5), (1.10) and the inequality $\left\|t^{-1}\left(D_{\ell}+i\right)^{-1}\right\| \leq 2$, which follows from (1.9), we have

$$
\begin{gathered}
\left\|\left(D_{\ell}+i\right)^{-1}\left[\chi_{(0, \delta / 4)} Q(D+i)^{-1}\right]^{k}\right\| \\
\leq \limsup _{\epsilon \downarrow 0}\left\|\left(D_{\ell}+i\right)^{-1} q^{1 / 2}\left[\chi_{(\epsilon, \delta / 4)} Q^{1 / 2}\left(D_{\ell}+i\right)^{-1} Q^{1 / 2} \chi_{(\epsilon, \delta / 4)}\right]^{k-1} q^{1 / 2}\left(D_{\ell}+i\right)^{-1}\right\| \\
\leq 4 \frac{(1+\ell-\delta)^{k}}{\left(1+\ell-\frac{\delta}{2}\right)^{k-1}} .
\end{gathered}
$$


Hence the series $R=\sum_{k=0}^{\infty}\left(D_{\ell}+i\right)^{-1}\left[q(D+i)^{-1}\right]^{k}$ converges in the operator norm and, by (1.12), $R-\left(D_{\ell}+i\right)^{-1}$ is a Hilbert-Schmidt operator.

It is easy to verify that if $\varphi \in C_{c}^{\infty}(0, \infty) \otimes \mathbf{C}^{2}$, then

$$
R\left(D_{\ell}-q+i\right) \varphi=\varphi \text {. }
$$

Hence if $D_{\ell}-q$ also denotes an arbitrary self-adjoint extension of the minimal operator, then $R-\left(D_{\ell}-q+i\right)^{-1}$ is an operator of finite rank. Thus $\left(D_{\ell}-q+i\right)^{-1}-$ $\left(D_{\ell}+i\right)^{-1}$ is a Hilbert-Schmidt operator. Since $Q=q+q_{1}$ where $q_{1}=Q \chi_{(\delta / 4, \infty)}$ is bounded, it now follows from Lemma 1.1 that $\left(D_{\ell}-Q+i\right)^{-1}-\left(D_{\ell}-q_{1}+i\right)^{-1}$ is a Hilbert-Schmidt operator. Since $Q$ is assumed to have a bounded support, it follows from Lemma 1.2 that $\left(D_{\ell}-q_{1}+i\right)^{-1}-\left(D_{\ell}+i\right)^{-1}=\left(D_{\ell}-q_{1}+i\right)^{-1} q_{1}\left(D_{\ell}+i\right)^{-1}$ is a Hilbert-Schmidt operator. This completes the proof.

Theorem 1.7. For any $z \in \mathbf{C} \backslash \mathbf{R}$ and for any self-adjoint extension of $D_{\ell}-V$,

$$
\left(D_{\ell}-z\right)^{-1}-\left(D_{\ell}-V-z\right)^{-1}
$$

is a Hilbert-Schmidt operator.

Proof. Let us first consider the case $\left|v\left(0^{+}\right)\right|>\sqrt{(1+\ell)^{2}-(1 / 4)}$. According to Lemma 1.5, $\left(D_{\ell}-V-z\right)^{-1} \chi_{(0, d+1)}$ is a Hilbert-Schmidt operator. $\left(D_{\ell}-z\right)^{-1} \chi_{(0, d+1)}$ is also a Hilbert-Schmidt operator by Lemma 1.2. Therefore it suffices to show that if $\eta$ is a $C^{\infty}$-function on $(0, \infty)$ such that $\eta=0$ on $(0, d+1 / 3)$ and $\eta=1$ on $(d+2 / 3, \infty)$, then

$$
\left[\left(D_{\ell}-z\right)^{-1}-\left(D_{\ell}-V-z\right)^{-1}\right] \eta
$$

is a Hilbert-Schmidt operator. Because $\eta V=0$, we have

$$
\begin{aligned}
{\left[\left(D_{\ell}-z\right)^{-1}-(\right.} & \left.\left.D_{\ell}-V-z\right)^{-1}\right] \eta\left(D_{\ell}-z\right) \\
= & \left(D_{\ell}-z\right)^{-1} \eta\left(D_{\ell}-z\right)-\left(D_{\ell}-V-z\right)^{-1} \eta\left(D_{\ell}-V-z\right) \\
= & \left(D_{\ell}-z\right)^{-1}\left(D_{\ell}-z\right) \eta-\left(D_{\ell}-V-z\right)^{-1}\left(D_{\ell}-V-z\right) \eta \\
& +\left(D_{\ell}-z\right)^{-1}\left[\eta, D_{\ell}-z\right]-\left(D_{\ell}-V-z\right)^{-1}\left[\eta, D_{\ell}-V-z\right] \\
= & \left\{\left(D_{\ell}-z\right)^{-1}-\left(D_{\ell}-V-z\right)^{-1}\right\}\left[\eta, D_{\ell}\right]\left(D_{\ell}-z\right)^{-1}\left(D_{\ell}-z\right) .
\end{aligned}
$$

Since $\left(D_{\ell}-z\right)$ has a dense range in $H$, we have

$$
\begin{aligned}
& {\left[\left(D_{\ell}-z\right)^{-1}-\left(D_{\ell}-V-z\right)^{-1}\right] \eta} \\
& \quad=\left\{\left(D_{\ell}-z\right)^{-1}-\left(D_{\ell}-V-z\right)^{-1}\right\}\left[\eta, D_{\ell}\right]\left(D_{\ell}-z\right)^{-1},
\end{aligned}
$$

which is a Hilbert-Schmidt operator because $\left[\eta, D_{\ell}\right]$ is a matrix-valued function with a bounded support.

In the case $\left|v\left(0^{+}\right)\right|<1+\ell$ it follows from Proposition 1.6 that there is a $\sigma>0$ such that $\left(D_{\ell}-V \chi_{(0, \sigma)}-z\right)^{-1}-\left(D_{\ell}-z\right)^{-1}$ is a Hilbert-Schmidt operator. Since $V \chi_{(\sigma, \infty)}$ is bounded, Lemma 1.1 tells us that $\left(D_{\ell}-V-z\right)^{-1}-\left(D_{\ell}-V \chi_{(\sigma, \infty)}-z\right)^{-1}$ is also a Hilbert-Schmidt operator. By Lemma 1.2 and the fact that the support of $V$ is bounded,

$$
\left(D_{\ell}-V \chi_{(\sigma, \infty)}-z\right)^{-1}-\left(D_{\ell}-z\right)^{-1}=\left(D_{\ell}-V \chi_{(\sigma, \infty)}-z\right)^{-1} V \chi_{(\sigma, \infty)}\left(D_{\ell}-z\right)^{-1}
$$

is a Hilbert-Schmidt operator. Hence so is $\left(D_{\ell}-V-z\right)^{-1}-\left(D_{\ell}-z\right)^{-1}$. 


\section{DEFICIENCY INDICES}

In this section $V(t)=v(t) / t$ will be exactly the same as in the previous section. But by $D_{\ell}-V$ we will now mean the minimal operator on the domain $C_{c}^{\infty}(0, \infty) \otimes$ $\mathbf{C}^{2}$. Recall that the deficiency indices of $D_{\ell}-V$ are either $(0,0)$ or $(1,1)$.

Proposition 2.1. Let $\ell \in \mathbf{Z}_{+}$and let $Q$ be a real-valued measurable function on $(0, \infty)$ such that $|Q(t)| \leq(\ell+(1 / 2)) / t$ for a.e. $t \in(0, \infty)$. Then $D_{\ell}-Q$ is essentially self-adjoint on $C_{c}^{\infty}(0, \infty) \otimes \mathbf{C}^{2}$.

Proof. We have

$$
t\left(D_{\ell}^{2}+1\right) t=-t \frac{d^{2}}{d t^{2}} t+\left[\begin{array}{cc}
(1+\ell)^{2} & 1+\ell \\
1+\ell & (1+\ell)^{2}
\end{array}\right]+t^{2} \geq-t \frac{d^{2}}{d t^{2}} t+(1+\ell) \ell .
$$

On the other hand, if $g \in C_{c}^{\infty}(0, \infty)$, then

$$
\begin{aligned}
\int_{0}^{\infty}\left|(t g)^{\prime}\right|^{2} d t & =\int_{0}^{\infty}\left|t g^{\prime}+g\right|^{2} d t=\int_{0}^{\infty}\left\{t^{2}\left|g^{\prime}\right|^{2}+\left(t|g|^{2}\right)^{\prime}\right\} d t \\
& =\int_{0}^{\infty} t^{2}\left|g^{\prime}\right|^{2} d t \geq \frac{1}{4} \int_{0}^{\infty}|g|^{2} d t
\end{aligned}
$$

Thus

$$
\left\|\left(D_{\ell}+i\right) t \varphi\right\|^{2}=\left\langle t\left(D_{\ell}^{2}+1\right) t \varphi, \varphi\right\rangle \geq\left(\frac{1}{4}+(1+\ell) \ell\right)\|\varphi\|^{2}=\left(\ell+\frac{1}{2}\right)^{2}\|\varphi\|^{2}
$$

for all $\varphi \in C_{c}^{\infty}(0, \infty) \otimes \mathbf{C}^{2}$. This implies

$$
\left\|\left(D_{\ell}+i\right) \psi\right\| \geq\left(\ell+\frac{1}{2}\right)\left\|t^{-1} \psi\right\| \geq\|Q \psi\|
$$

whenever $\psi \in C_{c}^{\infty}(0, \infty) \otimes \mathbf{C}^{2}$. This shows that $Q$ is $D_{\ell^{-}}$bounded with relative bound 1. Hence it follows from [6, Theorem V.4.6] that $D_{\ell}-Q$ is essentially self-adjoint on $C_{c}^{\infty}(0, \infty) \otimes \mathbf{C}^{2}$.

Recall that $V_{b}(t)=b \chi_{(0, d)}(t) / t$ on $(0, \infty)$. If $(1+\ell)^{2}-b^{2} \geq 1 / 4$, then the operator $D_{\ell}-V_{b}$ is known to be essentially self-adjoint on $C_{c}^{\infty}(0, \infty) \otimes \mathbf{C}^{2}$. This result, which is usually stated in terms of the limit point-limit circle alternatives, goes at least as far back as [12]. (Also see [4].) There are many different proofs using differential equation techniques. We would like to sketch an alternate proof by counting the deficiency indices of the minimal operator.

Consider the operator $D_{\ell}-V_{b}$ on $C_{c}^{\infty}(0, R) \otimes \mathbf{C}^{2}$ where $R>d$. To show that $D_{\ell}-V_{b}$ is essentially self-adjoint on $C_{c}^{\infty}(0, \infty) \otimes \mathbf{C}^{2}$, it suffices to show that on $C_{c}^{\infty}(0, R) \otimes \mathbf{C}^{2}$, the same operator has deficiency indices $(1,1)$ in $L^{2}(0, R) \otimes \mathbf{C}^{2}$. This is because we know that on the domain $C_{c}^{\infty}(R, \infty) \otimes \mathbf{C}^{2}, D_{\ell}-V_{b}$ has deficiency indices $(1,1)$ in $L^{2}(R, \infty) \otimes \mathbf{C}^{2}$. If $\mathcal{D}_{\ell, b, R}$ and $\mathcal{D}_{\ell, b}$ denote the domains for the closures of $D_{\ell}-V_{b}$ on $C_{c}^{\infty}(0, R) \otimes \mathbf{C}^{2}+C_{c}^{\infty}(R, \infty) \otimes \mathbf{C}^{2}$ and on $C_{c}^{\infty}(0, \infty) \otimes \mathbf{C}^{2}$ respectively, then $\operatorname{dim}\left(\mathcal{D}_{\ell, b} / \mathcal{D}_{\ell, b, R}\right)=2$. Therefore the desired essential self-adjointness follows from the assertion about the deficiency indices of $D_{\ell}-V_{b}$ on $C_{c}^{\infty}(0, R) \otimes \mathbf{C}^{2}$.

By (1.1), in the case $(1+\ell)^{2}-b^{2} \geq 1 / 4$, there is, up to constant multiples, only one non-trivial solution of the $2 \times 2$ system $\left(D_{\ell}-V_{b}\right) u=0$ that belongs to $L^{2}(0, R) \otimes \mathbf{C}^{2}$. If the deficiency indices of $D_{\ell}-V_{b}$ on $C_{c}^{\infty}(0, R) \otimes \mathbf{C}^{2}$ were not $(1,1)$, then 0 would not belong to the domain of regularity for the symmetric operator $D_{\ell}-V_{b}$ on $C_{c}^{\infty}(0, R) \otimes \mathbf{C}^{2}$ (see pages 37 and 42 of [7]). According to Corollary 
14.10.3 of [7], there are only two possible scenarios when this happens: Either 0 is an eigenvalue of the closure of $D_{\ell}-V_{b}$ on $C_{c}^{\infty}(0, R) \otimes \mathbf{C}^{2}$ or 0 belongs to the continuous spectrum of every self-adjoint extension of this operator.

But, because $D_{\ell}-V_{b}$ is regular at $R$, if $u$ belongs to the domain of the closure of $D_{\ell}-V_{b}$ on $C_{c}^{\infty}(0, R) \otimes \mathbf{C}^{2}$ and satisfies the differential equation $\left(D_{\ell}-V_{b}\right) u=0$, then $u=0$. Hence the first scenario cannot occur. On the other hand, no self-adjoint extension of $D_{\ell}-V_{b}$ on $C_{c}^{\infty}(0, R) \otimes \mathbf{C}^{2}$ has any continuous spectrum. For, if $A$ and $B$ are self-adjoint extensions of $\left(D_{\ell}-V_{b}\right) \mid C_{c}^{\infty}(0, R) \otimes \mathbf{C}^{2}$ and $\left(D_{\ell}-V_{b}\right) \mid C_{c}^{\infty}(0, \infty) \otimes \mathbf{C}^{2}$ in $L^{2}(0, R) \otimes \mathbf{C}^{2}$ and in $H=L^{2}(0, \infty) \otimes \mathbf{C}^{2}$ respectively, and if we regard $A$ as an operator on $H$ by setting $A=0$ on $L^{2}(R, \infty) \otimes \mathbf{C}^{2}$, then the operators $(A-z)^{-1}$ and $(B-z)^{-1}$ agree on $\left(D_{\ell}-V_{b}\right) C_{c}^{\infty}(0, R) \otimes \mathbf{C}^{2}$ when $z \in \mathbf{C} \backslash \mathbf{R}$. Thus the operator $(A-z)^{-1}-(B-z)^{-1}$ has a finite rank when restricted to $L^{2}(0, R) \otimes \mathbf{C}^{2}$. By Theorem 1.7 and Lemma $1.2,(B-z)^{-1} \chi_{(0, R)}$ is a Hilbert-Schmidt operator. Hence $A$ has no continuous spectrum. Thus $D_{\ell}-V_{b}$ is essentially self-adjoint on $C_{c}^{\infty}(0, \infty) \otimes \mathbf{C}^{2}$.

We would like to mention that in the case $(1+\ell)^{2}-b^{2} \geq 1 / 4$ and $\sqrt{(1+\ell)^{2}-b^{2}}$ is not an integer, the easiest way to show that $D_{\ell}-V_{b}$ is essentially self-adjoint on $C_{c}^{\infty}(0, \infty) \otimes \mathbf{C}^{2}$ is to solve the equation $\left(D_{\ell}-V_{b}-z\right) u=0$ using (1.1) and Laurent series in $t$. This, however, does not work if $\sqrt{(1+\ell)^{2}-b^{2}}$ is an integer.

This discussion prepares us for the main result of this section.

Theorem 2.2. (a) If $\left|v\left(0^{+}\right)\right|<\sqrt{(1+\ell)^{2}-(1 / 4)}$, then $D_{\ell}-V$ is essentially selfadjoint on $C_{c}^{\infty}(0, \infty) \otimes \mathbf{C}^{2}$.

(b) If $\left|v\left(0^{+}\right)\right|>\sqrt{(1+\ell)^{2}-(1 / 4)}$, then on $C_{c}^{\infty}(0, \infty) \otimes \mathbf{C}^{2}$ the deficiency indices of $D_{\ell}-V$ are $(1,1)$.

Remark 1. This theorem clearly suggests that, if $\left|v\left(0^{+}\right)\right|=\sqrt{(1+\ell)^{2}-(1 / 4)}$, the deficiency indices of $D_{\ell}-V$ in general cannot be determined without further assumptions on $v$.

Remark 2. An immediate implication of part (a) is that for such a pair of $v$ and $\ell$, the differential operator $D_{\ell}-V$ is in the limit point case at 0 . The reader should compare part (a) with [4, Theorem 2], which is a similar result stated in terms of the limit point-limit circle alternatives. Even though [4] treats a slightly larger class of differential operators, part (a) is a stronger result in the respect that $v$ is required to have a limit only at 0 and nowhere else. The most significant difference between part (a) and [4], however, lies in the proof. While [4] uses exclusively differential equation techniques, we rely on operator theory, which, in our opinion, is a simpler approach.

We need some technical preparations for the proof of Theorem 2.2. We introduced the bounded operator

$$
\left(T_{\mu} f\right)(t)=t^{-\mu-1} \int_{0}^{t} s^{\mu} f(s) d s
$$

for $\mu>-1 / 2$ in Section 1. Since $L^{2}(0, d)$ is an invariant subspace for $T_{\mu}$, we may consider it as an operator on $L^{2}(0, d)$ as well. Let us define

$$
\left(S_{\mu} f\right)(t)=t^{\mu-1} \int_{t}^{d} s^{-\mu} f(s) d s
$$

on $L^{2}(0, d)$. For $\mu>1 / 2$, this operator is also bounded according to Hardy's inequality [1, Lemma 3.3.9]. 
Proposition 2.3. Suppose $\ell \in \mathbf{Z}_{+}$and $b \in \mathbf{R}$ are such that $(1+\ell)^{2}-b^{2} \neq 1 / 4$. Let $\mathcal{D}_{\ell, b}$ denote the domain of the closure of $D_{\ell}-V_{b}$ on $C_{c}^{\infty}(0, \infty) \otimes \mathbf{C}^{2}$. Then for every $\varphi \in \mathcal{D}_{\ell, b}$, the function $t^{-1} \varphi(t)$ belongs to $L^{2}(0, \infty) \otimes \mathbf{C}^{2}$.

Proof. Let $\varphi \in \mathcal{D}_{b, \ell}$ and $\eta \in C_{c}^{\infty}(\mathbf{R})$. We claim that $\eta \varphi \in \mathcal{D}_{\ell, b}$. This is because if $\left\{\varphi_{n}\right\}$ is a sequence in $C_{c}^{\infty}(0, \infty) \otimes \mathbf{C}^{2}$ with $\left\|\varphi-\varphi_{n}\right\| \rightarrow 0$ and $\left\|\left(D_{\ell}-V_{b}\right)\left(\varphi-\varphi_{n}\right)\right\| \rightarrow$ 0 , then

$$
\left\|\left(D_{\ell}-V_{b}\right) \eta\left(\varphi-\varphi_{n}\right)\right\| \leq\left\|\eta\left(D_{\ell}-V_{b}\right)\left(\varphi-\varphi_{n}\right)\right\|+\left\|\left[D_{\ell}, \eta\right]\left(\varphi-\varphi_{n}\right)\right\| \rightarrow 0 .
$$

Thus to prove the proposition, we only need to consider $\varphi \in \mathcal{D}_{\ell, b}$ whose support is contained in, say, $(0, d)$.

First let us consider the case $(1+\ell)^{2}-b^{2}>1 / 4$. Let $\nu=1+\ell-b$ and $\mu=\sqrt{(1+\ell)^{2}-b^{2}}$. Then $\mu>1 / 2$ by our assumption. Recall that the linear system $\left(D_{\ell}-V_{b}\right) u=0$ has a fundamental solution matrix $\Phi_{b, \ell}$ given by (1.1) for $t \in(0, d)$. Accordingly,

$$
\Phi_{b, \ell}^{-1}(t)=\frac{1}{2 \nu \mu}\left[\begin{array}{cc}
\nu t^{-\mu} & -\mu t^{-\mu} \\
\nu t^{\mu} & \mu t^{\mu}
\end{array}\right]
$$

on the same interval. One solves the equation $\left(D_{\ell}-V_{b}\right) u=h$ by setting $u=\Phi_{b, \ell} c$ and solving $B \Phi_{b, \ell} c^{\prime}=h$. Thus $c^{\prime}=\Phi_{b, \ell}^{-1} B^{-1} h$. If we write

$$
h=\left[\begin{array}{l}
h_{1} \\
h_{2}
\end{array}\right] \text { and } c=\left[\begin{array}{l}
c_{1} \\
c_{2}
\end{array}\right] \text {, }
$$

then we may take

$$
c_{1}(t)=\frac{1}{2 \nu \mu} \int_{t}^{d} s^{-\mu}\left(\nu h_{2}(s)+\mu h_{1}(s)\right) d s
$$

and

$$
c_{2}(t)=\frac{1}{2 \nu \mu} \int_{0}^{t} s^{\mu}\left(-\nu h_{2}(s)+\mu h_{1}(s)\right) d s .
$$

Hence if we define

$$
F(h)(t)=\frac{t}{2 \nu \mu}\left[\begin{array}{c}
\mu\left[S_{\mu}\left(\nu h_{2}+\mu h_{1}\right)\right](t)+\mu\left[T_{\mu}\left(-\nu h_{2}+\mu h_{1}\right)\right](t) \\
-\nu\left[S_{\mu}\left(\nu h_{2}+\mu h_{1}\right)\right](t)+\nu\left[T_{\mu}\left(-\nu h_{2}+\mu h_{1}\right)\right](t)
\end{array}\right],
$$

then $u=F(h)$ is a solution of the inhomogeneous system $\left(D_{\ell}-V_{b}\right) u=h$.

Let $\varphi \in \mathcal{D}_{\ell, b}$ be given and suppose that its support is contained in $(0, d)$. Let $\tilde{D}$ denote the closure of $D_{\ell}-V_{b}$ and let $h=\tilde{D} \varphi$. Obviously the support of $h$ is contained in $(0, d)$. Now both $\varphi$ and $F(h)$ solve the differential equation $\left(D_{\ell}-V_{b}\right) u=h$ on $(0, d)$. Therefore $\varphi-F(h)$ solves the homogeneous equation $\left(D_{\ell}-V_{b}\right) u=0$ on the same interval. Since both $\varphi$ and $F(h)$ belong to $L^{2}(0, d) \otimes \mathbf{C}^{2}$, by (1.1), there is an $a_{0} \in \mathbf{C}$ such that

$$
(\varphi-F(h))(t)=a_{0}\left[\begin{array}{c}
\mu t^{\mu} \\
-\nu t^{\mu}
\end{array}\right] .
$$

By the $L^{2}$-boundedness of $T_{\mu}$ and $S_{\mu}, t^{-1} F(h)$ belongs $L^{2}(0, d) \otimes \mathbf{C}^{2}$. Therefore so does $t^{-1} \varphi$.

For the case $(1+\ell)^{2}-b^{2}<1 / 4$, we need the integral operator $R$ defined in the proof of Lemma 1.5. Recall that

$$
\varphi=R\left(D_{\ell}-V_{b}-z\right) \varphi \text { whenever } \varphi \in C_{c}^{\infty}(0, \infty) \otimes \mathbf{C}^{2} .
$$


Since the operator $\chi_{(0, c)} R \chi_{(0, c)}$ is bounded when $0<c<\infty$, a routine argument yields $\psi=R\left(D_{\ell}-V_{b}-z\right) \psi$ for every $\psi \in \mathcal{D}_{\ell, b}$ with bounded support. Lemma 1.4 tells us that $\chi_{(0, c)} t^{-1} R \chi_{(0, c)}$ is a bounded operator. Hence $t^{-1} \psi \in L^{2}(0, \infty) \otimes$ $\mathbf{C}^{2}$.

Proof of Theorem 2.2. (a) Let $b=v\left(0^{+}\right)$. Our assumption says $(1+\ell)^{2}-b^{2}>$ $1 / 4$. Let $z \in \mathbf{C} \backslash \mathbf{R}$ be fixed. We know that $D_{\ell}-V_{b}$ is essentially self-adjoint on $C_{c}^{\infty}(0, \infty) \otimes \mathbf{C}^{2}$. By Proposition 2.3 and the uniform boundedness principle, there is an $M>0$ such that

$$
\left\|\chi_{(\epsilon, \infty)} t^{-1}\left(D_{\ell}-V_{b}-z\right)^{-1}\right\| \leq M
$$

for every $\epsilon>0$. Since $b=v\left(0^{+}\right)=\lim _{t \downarrow 0} v(t)$, there is a $\delta \in(0, d)$ such that

$$
\left\|(v-b) \chi_{(0, \delta)}\right\|_{\infty} M \leq 1 / 2 .
$$

Proposition 2.3 also tells us that the strong operator limit

$$
T=\lim _{\epsilon \downarrow 0}\left(V-V_{b}\right) \chi_{(\epsilon, \delta)}\left(D_{\ell}-V_{b}-z\right)^{-1}
$$

exists and $\|T\| \leq 1 / 2$. In particular $1-T$ is an invertible operator.

If $\varphi \in C_{c}^{\infty}(0, \infty) \otimes \mathbf{C}^{2}$, then

$$
(1-T)\left(D_{\ell}-V_{b}-z\right) \varphi=\left(D_{\ell}-V_{b}-z-\left(V-V_{b}\right) \chi_{(0, \delta)}\right) \varphi .
$$

Since $\left(D_{\ell}-V_{b}-z\right) C_{c}^{\infty}(0, \infty) \otimes \mathbf{C}^{2}$ is dense in $H$ and $1-T$ is invertible, $\left(D_{\ell}-V_{b}-z-\left(V-V_{b}\right) \chi_{(0, \delta)}\right) C_{c}^{\infty}(0, \infty) \otimes \mathbf{C}^{2}$ is also dense in $H$. Hence the operator $D_{\ell}-V_{b}-\left(V-V_{b}\right) \chi_{(0, \delta)}$ is essentially self-adjoint on $C_{c}^{\infty}(0, \infty) \otimes \mathbf{C}^{2}$. But $V_{b}+\left(V-V_{b}\right) \chi_{(0, \delta)}$ differs from $V$ by a bounded function. Therefore $D_{\ell}-V$ is also essentially self-adjoint on $C_{c}^{\infty}(0, \infty) \otimes \mathbf{C}^{2}$.

(b) Again, write $b$ for $v\left(0^{+}\right)$. Then $(1+\ell)^{2}-b^{2}<1 / 4$. We now let

$$
(R g)(t)=\Psi_{b, \ell, i}(t) \int_{0}^{t} \Psi_{b, \ell, i}^{-1}(s) B^{-1} g(s) d s
$$

where, as we recall, $\Psi_{b, \ell, i}$ is the fundamental solution matrix of the linear system $\left(D_{\ell}-V_{b}-i\right) u=0$. Fix a $c \in(0, \infty)$. Lemma 1.4 tells us that $\chi_{(0, c)} t^{-1} R \chi_{(0, c)}$ is a bounded operator. The identity $R\left(D_{\ell}-V_{b}-i\right) \varphi=\varphi$ holds whenever $\varphi \in$ $C_{c}^{\infty}(0, c) \otimes \mathbf{C}^{2}$. Let $\delta>0$ be such that $\left\|\left(V-V_{b}\right) \chi_{(0, \delta)} R \chi_{(0, c)}\right\| \leq 1 / 2$.

For every $\varphi \in C_{c}^{\infty}(0, c) \otimes \mathbf{C}^{2}$

$$
\left(D_{\ell}-V_{b}-\left(V-V_{b}\right) \chi_{(0, \delta)}-i\right) \varphi=\left(1-\left(V-V_{b}\right) \chi_{(0, \delta)} R\right)\left(D_{\ell}-V_{b}-i\right) \varphi .
$$

By Lemma 1.3, the orthogonal complement of $\left(D_{\ell}-V_{b}-i\right) C_{c}^{\infty}(0, c) \otimes \mathbf{C}^{2}$ in $L^{2}(0, c) \otimes$ $\mathbf{C}^{2}$ has dimension 2. Therefore, on the domain $C_{c}^{\infty}(0, c) \otimes \mathbf{C}^{2}$, the deficiency indices of $D_{\ell}-V_{b}-\left(V-V_{b}\right) \chi_{(0, \delta)}$ in $L^{2}(0, c) \otimes \mathbf{C}^{2}$ are $(2,2)$. But this operator differs from $D_{\ell}-V$ by a bounded operator. Hence, on $C_{c}^{\infty}(0, c) \otimes \mathbf{C}^{2}$, the deficiency indices of $D_{\ell}-V$ in $L^{2}(0, c) \otimes \mathbf{C}^{2}$ are also $(2,2)$.

Thus every solution of the linear system $\left(D_{\ell}-V-i\right) u=0$ is square-integrable near 0 . We know what happens near $\infty$. Therefore there is, up to scalar multiples, exactly one non-trivial solution $u_{+}$of this system which belongs to $L^{2}(0, \infty) \otimes$ $\mathbf{C}^{2}$. Similarly there is exactly one non-trivial $u_{-}$which solves the linear system 
$\left(D_{\ell}-V+i\right) u=0$ and which belongs to $L^{2}(0, \infty) \otimes \mathbf{C}^{2}$. It follows from integration by parts that

$$
\left\langle\left(D_{\ell}-V\right) \varphi, u_{ \pm}\right\rangle= \pm i\left\langle\varphi, u_{ \pm}\right\rangle
$$

whenever $\varphi \in C_{c}^{\infty}(0, \infty) \otimes \mathbf{C}^{2}$. Hence the deficiency indices of $D_{\ell}-V$ are $(1,1)$.

\section{The Decomposition of $\sigma \cdot p$}

Recall that the Pauli spin matrices are usually written as

$$
\sigma_{1}=\left[\begin{array}{ll}
0 & 1 \\
1 & 0
\end{array}\right], \quad \sigma_{2}=\left[\begin{array}{cc}
0 & -i \\
i & 0
\end{array}\right], \quad \sigma_{3}=\left[\begin{array}{cc}
1 & 0 \\
0 & -1
\end{array}\right] .
$$

Let $\left(p_{1}, p_{2}, p_{3}\right)=\left(-i \partial / \partial x_{1},-i \partial / \partial x_{2},-i \partial / \partial x_{3}\right)$ and denote

$$
\sigma \cdot p=p_{1} \sigma_{1}+p_{2} \sigma_{2}+p_{3} \sigma_{3}
$$

We denote the angular momentum operators with respect the three coordinate axes by

$$
\Lambda_{1}=x_{2} p_{3}-x_{3} p_{2}, \quad \Lambda_{2}=x_{3} p_{1}-x_{1} p_{3}, \quad \Lambda_{3}=x_{1} p_{2}-x_{2} p_{1}
$$

Let

$$
\Lambda=\Lambda_{1} \sigma_{1}+\Lambda_{2} \sigma_{2}+\Lambda_{3} \sigma_{3} \text { and } L=\Lambda_{1}^{2}+\Lambda_{2}^{2}+\Lambda_{3}^{2}
$$

Because of the commutation relations $\left[\Lambda_{1}, \Lambda_{2}\right]=i \Lambda_{3}$ etc. and the commutation relations between the Puali spin matrices, the relation between $L$ and $\Lambda$ is

$$
L=\Lambda^{2}+\Lambda \text {. }
$$

Throughout the remainder of the paper, $r$ stands for $|x|=\left(x_{1}^{2}+x_{2}^{2}+x_{3}^{2}\right)^{1 / 2}$. Let $\sigma_{\rho}$ denote the multiplication operator $r^{-1}\left(x_{1} \sigma_{1}+x_{2} \sigma_{2}+x_{3} \sigma_{3}\right)$. The above operators are defined on the appropriate dense domains in $L^{2}\left(\mathbf{R}^{3}\right) \otimes \mathbf{C}^{2}$. We will say more about these domains later.

The operators $\Lambda_{1}, \Lambda_{2}, \Lambda_{3}$ commute with the multiplication by any radial function. In addition, $\left[\Lambda_{j}, x_{j}\right]=0, j=1,2,3$. It is easy to see that

$$
\left[\Lambda_{1} x_{1}+\Lambda_{2} x_{2}+\Lambda_{3} x_{3}\right] r^{-1}=r^{-1}\left[x_{1} \Lambda_{1}+x_{2} \Lambda_{2}+x_{3} \Lambda_{3}\right]=0 .
$$

Combining this with the well-known identities

$$
\sigma_{1} \sigma_{2}=i \sigma_{3}, \quad \sigma_{2} \sigma_{3}=i \sigma_{1}, \quad \sigma_{3} \sigma_{1}=i \sigma_{2}
$$

and

$$
\left[\Lambda_{1}, x_{2}\right]=\left[x_{1}, \Lambda_{2}\right]=i x_{3}, \quad\left[\Lambda_{3}, x_{1}\right]=\left[x_{3}, \Lambda_{1}\right]=i x_{2}, \quad\left[\Lambda_{2}, x_{3}\right]=\left[x_{2}, \Lambda_{3}\right]=i x_{1},
$$

we obtain

$$
\Lambda \sigma_{\rho}=-\sigma_{\rho}(\Lambda+2) \text {. }
$$

This identity along with (3.1) will play a key role in our analysis of the Dirac operator.

We write the spherical coordinates in $\mathbf{R}^{3}$ as $x_{3}=r \cos \gamma, x_{1}=r \sin \gamma \cos \lambda$, $x_{2}=r \sin \gamma \sin \lambda$. For any $\ell \in \mathbf{N}$ and $-\ell \leq m \leq \ell$, we have the (unnormalized) spherical harmonics

$$
X_{\ell}^{m}(\gamma, \lambda)=e^{i m \lambda} P_{\ell}^{|m|}(\cos \gamma)
$$


where

$$
P_{\ell}^{k}(t)=\left(1-t^{2}\right)^{k / 2} \frac{d^{k+\ell}\left(1-t^{2}\right)^{\ell}}{d t^{k+\ell}}
$$

We also set $X_{0}^{0}=1$. Let us write the usual spin vectors as

Let

$$
s_{1 / 2}=\left[\begin{array}{l}
1 \\
0
\end{array}\right] \text { and } s_{-1 / 2}=\left[\begin{array}{l}
0 \\
1
\end{array}\right]
$$

$$
\mathcal{S}=\text { the linear span of }\left\{X_{\ell}^{m} s_{j}: \ell \in \mathbf{Z}_{+}, m \in \mathbf{Z},|m| \leq \ell, j= \pm 1 / 2\right\} .
$$

As usual, $\mathcal{S}$ is endowed with the inner product induced by the area measure on the unit sphere $S^{2}$ :

$$
\langle f, g\rangle=\int_{0}^{\pi} \sin \gamma\left[\int_{0}^{2 \pi}\langle f(\gamma, \lambda), g(\gamma, \lambda)\rangle_{\mathbf{C}^{2}} d \lambda\right] d \gamma .
$$

Thus $\left\langle X_{\ell}^{m} s_{j}, X_{l^{\prime}}^{m^{\prime}} s_{j^{\prime}}\right\rangle=0$ if $(m, \ell, j) \neq\left(m^{\prime}, \ell^{\prime}, j^{\prime}\right)$.

We know that $L$ can be regarded as an operator on $\mathcal{S}$. Indeed $L X_{\ell}^{m} s_{j}=$ $\ell(\ell+1) X_{\ell}^{m} s_{j}$. Let us verify that $\sigma_{\rho}$ also maps $\mathcal{S}$ to itself. Indeed, in spherical coordinates,

$$
\sigma_{\rho}=\left[\begin{array}{cc}
\cos \gamma & \sin \gamma e^{-i \lambda} \\
\sin \gamma e^{i \lambda} & -\cos \gamma
\end{array}\right]
$$

It suffices to verify that, for any $X_{\ell}^{m}, \cos \gamma X_{\ell}^{m}$ and $\sin \gamma e^{ \pm i \lambda} X_{\ell}^{m}$ are linear combinations of spherical harmonics. Now $\cos \gamma X_{\ell}^{m}=e^{i m \lambda} \sin ^{|m|} \gamma Q(\cos \gamma)$, where $Q$ is a polynomial. Therefore $\cos \gamma X_{\ell}^{m}$ has the desired property. On the other hand

$$
\sin \gamma e^{i \lambda} \cdot \sin ^{|m|} \gamma e^{i m \lambda} P(\cos \gamma)=\sin ^{|m|+1} \gamma e^{i(m+1) \lambda} P(\cos \gamma) .
$$

We can write $\sin ^{|m|+1} \gamma$ as $\sin ^{|m+1|} \gamma$ or $\sin ^{|m+1|} \gamma\left(1-\cos ^{2} \gamma\right)$ depending on whether $m \geq 0$ or $m \leq-1$. This shows that $\sin \gamma e^{i \lambda} X_{\ell}^{m}$ is a linear combination of spherical harmonics. The case of $\sin \gamma e^{-i \lambda} X_{\ell}^{m}$ can be treated similarly. Therefore $\sigma_{\rho}$ maps $\mathcal{S}$ to itself.

Let

$$
\Lambda_{+}=\Lambda_{1}+i \Lambda_{2}=e^{i \lambda}(\partial / \partial \gamma+i \cot \gamma \partial / \partial \lambda)
$$

and

$$
\Lambda_{-}=\Lambda_{1}-i \Lambda_{2}=e^{-i \lambda}(-\partial / \partial \gamma+i \cot \gamma \partial / \partial \lambda) .
$$

Then $\Lambda_{-} \Lambda_{+}=L-\left(\Lambda_{3}^{2}+\Lambda_{3}\right)$ and $\Lambda_{+} \Lambda_{-}=L-\left(\Lambda_{3}^{2}-\Lambda_{3}\right)$. Using spherical coordinates and the obvious fact that $\Lambda_{3} X_{\ell}^{m}=m X_{\ell}^{m}$, one easily verifies

$$
\begin{aligned}
\Lambda_{+} X_{\ell}^{m-1} & =-X_{\ell}^{m} \text { if } 0 \leq m-1 \leq \ell-1 ; \\
\Lambda_{+} X_{\ell}^{m-1} & =(m(m-1)-\ell(\ell+1)) X_{\ell}^{m} \quad \text { if } \quad-\ell \leq m-1 \leq-1 ; \\
\Lambda_{+} X_{\ell}^{\ell} & =\Lambda_{-} X_{\ell}^{-\ell}=0 ; \\
\Lambda_{-} X_{\ell}^{m} & =-X_{\ell}^{m-1} \text { if }-\ell+1 \leq m \leq 0 ; \\
\Lambda_{-} X_{\ell}^{m} & =(m(m-1)-\ell(\ell+1)) X_{\ell}^{m-1} \text { if } 1 \leq m \leq \ell ;
\end{aligned}
$$

$\ell \in \mathbf{N}$. Hence $\Lambda$ also maps $\mathcal{S}$ to itself.

We have the algebraic decomposition

$$
\mathcal{S}=\mathcal{S}_{0}+\mathcal{S}_{1}+\cdots+\mathcal{S}_{\ell}+\ldots
$$


where

$$
\mathcal{S}_{\ell}=\text { the linear span of }\left\{X_{\ell}^{m} s_{j}: j= \pm 1 / 2,|m| \leq \ell\right\}, \quad \ell \in \mathbf{Z}_{+},
$$

are orthogonal to each other. Since $\mathcal{S}_{\ell}$ is the kernel of the operator $L-\ell(\ell+1)$ and $\Lambda$ commutes with $L$, each $\mathcal{S}_{\ell}$ is also invariant under $\Lambda$. Furthermore, it follows from (3.1) that the only possible eigenvalues for $\Lambda$ on $\mathcal{S}_{\ell}$ are $\ell$ and $-\ell-1$ in the case $\ell \geq 1$. Obviously $L=\Lambda=0$ on $\mathcal{S}_{0}$. For each $\ell \in \mathbf{N}$, let $\mathcal{S}_{\ell}^{+}$and $\mathcal{S}_{\ell}^{-}$be the kernels of $\Lambda-\ell$ and $\Lambda+\ell+1$ on $\mathcal{S}_{\ell}$ respectively. Then $\mathcal{S}_{\ell}=\mathcal{S}_{\ell}^{+} \oplus \mathcal{S}_{\ell}^{-}$.

Proposition 3.1. We have

$$
\sigma_{\rho} \mathcal{S}_{0}=\mathcal{S}_{1}^{-} \quad \text { and } \sigma_{\rho} \mathcal{S}_{\ell}^{+}=\mathcal{S}_{\ell+1}^{-} \quad \text { for } \quad \ell \geq 1
$$

Moreover,

$$
\operatorname{dim}\left(\mathcal{S}_{\ell}^{-}\right)=2 \ell \text { and } \operatorname{dim}\left(\mathcal{S}_{\ell}^{+}\right)=2 \ell+2
$$

for every $\ell \in \mathbf{N}$.

Proof. We use induction. The equalities $\sigma_{\rho} \mathcal{S}_{0}=\mathcal{S}_{1}^{-}$and $\sigma_{\rho} \mathcal{S}_{\ell}^{+}=\mathcal{S}_{\ell+1}^{-}$follow immediately from $(3.2)$. Since $\operatorname{dim}\left(\mathcal{S}_{0}\right)=2$, it follows that $\operatorname{dim}\left(\mathcal{S}_{1}^{-}\right)=2$. On the other hand $\operatorname{dim}\left(\mathcal{S}_{1}\right)=2(2 \cdot 1+1)=6$. Therefore $\operatorname{dim}\left(\mathcal{S}_{1}^{+}\right)=4=2 \cdot 1+2$. Suppose that $\operatorname{dim}\left(\mathcal{S}_{\ell_{0}}^{-}\right)=2 \ell_{0}$ and $\operatorname{dim}\left(\mathcal{S}_{\ell_{0}}^{+}\right)=2 \ell_{0}+2$. Then $\operatorname{dim}\left(\mathcal{S}_{\ell_{0}+1}^{-}\right)=\operatorname{dim}\left(\sigma_{\rho} \mathcal{S}_{\ell_{0}}^{+}\right)=$ $2 \ell_{0}+2=2\left(\ell_{0}+1\right)$. Because $\operatorname{dim}\left(\mathcal{S}_{\ell+1}\right)=2(2(\ell+1)+1)=4(\ell+1)+2$ and $\mathcal{S}_{\ell}=\mathcal{S}_{\ell}^{+} \oplus \mathcal{S}_{\ell}^{-}$, we have $\operatorname{dim}\left(\mathcal{S}_{\ell_{0}+1}^{+}\right)=2\left(\ell_{0}+1\right)+2$.

Because $\sigma_{\rho}^{2}=1$, Proposition 3.1 allows us to write

$$
\begin{aligned}
\mathcal{S} & =\left(\mathcal{S}_{0}^{+} \oplus \sigma_{\rho} \mathcal{S}_{0}^{+}\right)+\left(\mathcal{S}_{1}^{+} \oplus \sigma_{\rho} \mathcal{S}_{1}^{+}\right)+\cdots+\left(\mathcal{S}_{\ell}^{+} \oplus \sigma_{\rho} \mathcal{S}_{\ell}^{+}\right)+\ldots \\
& =\left(\sigma_{\rho} \mathcal{S}_{1}^{-} \oplus \mathcal{S}_{1}^{-}\right)+\left(\sigma_{\rho} \mathcal{S}_{2}^{-} \oplus \mathcal{S}_{2}^{-}\right)+\cdots+\left(\sigma_{\rho} \mathcal{S}_{\ell}^{-} \oplus \mathcal{S}_{\ell}^{-}\right)+\ldots
\end{aligned}
$$

where $\mathcal{S}_{0}^{+}=\mathcal{S}_{0}$.

Lemma 3.2. Let $Q$ be a real-valued, measurable, radial function on $\mathbf{R}^{3}$. Suppose that there is a positive number $M$ such that $|Q(r)| \leq M / r$ a.e. on $\mathbf{R}^{3}$. Then the closure of the symmetric operator $\sigma \cdot p-Q$ on $C_{c}^{\infty}\left(\mathbf{R}^{3} \backslash\{0\}\right)$ is the same as its closure on $C_{c}^{\infty}\left(\mathbf{R}^{3}\right)$.

Proof. Let $\eta$ be a $C^{\infty}$-function on $[0, \infty)$ such that $\eta=1$ on $[1, \infty)$ and $\eta=0$ on $[0,1 / 2]$. Let $\eta_{n}(r)=\eta(n r), n=1,2, \ldots$. If $f \in C_{c}^{\infty}\left(\mathbf{R}^{3}\right)$, then $\eta_{n} f \in C_{c}^{\infty}\left(\mathbf{R}^{3} \backslash\{0\}\right)$ for every $n$ and $\lim _{n \rightarrow \infty}\left\|\eta_{n} f-f\right\|=0$. Since $Q f \in L^{2}\left(\mathbf{R}^{3}\right) \otimes \mathbf{C}^{2}$, we have $\lim _{n \rightarrow \infty}\left\|Q \eta_{n} f-Q f\right\|=0$. Therefore it suffices to show $\lim _{n \rightarrow \infty}\left\|\sigma \cdot p\left(\eta_{n} f-f\right)\right\|$ $=0$. But if we think of $\eta_{n}$ as a multiplication operator on $L^{2}\left(\mathbf{R}^{3}\right) \otimes \mathbf{C}^{2}$, then $\sigma \cdot p \eta_{n} f=\eta_{n} \sigma \cdot p f+\left[\sigma \cdot p, \eta_{n}\right] f$. Since $\left\|\eta_{n} \sigma \cdot p f-\sigma \cdot p f\right\| \rightarrow 0$, it suffices to show that $\left\|\left[\sigma \cdot p, \eta_{n}\right] f\right\| \rightarrow 0$.

Clearly $\left[p_{j}, \eta_{n}\right]$ is the operator of multiplication by $-i x_{j} \eta_{n}^{\prime}(r) / r=-i n x_{j} \eta^{\prime}(n r) / r$. Thus there is a $C>0$ such that $\left\|\left[\sigma \cdot p, \eta_{n}\right] f\right\|_{\infty} \leq C n$ for every $n \in \mathbf{N}$. On the other hand, the support of $\left[\sigma \cdot p, \eta_{n}\right]$ is contained in $\left\{x \in \mathbf{R}^{3}:|x| \leq 1 / n\right\}$. Hence $\left\|\left[\sigma \cdot p, \eta_{n}\right] f\right\|^{2} \leq C^{2} n^{2} m_{3}\left(\left\{x \in \mathbf{R}^{3}:|x| \leq 1 / n\right\}\right)=4 \pi C^{2} / 3 n \rightarrow 0$.

For each $\ell \in \mathbf{Z}_{+}$, let

$$
\Omega_{\ell}=\text { the linear span of }\left\{Y g: Y \in \mathcal{S}_{\ell}^{+} \oplus \sigma_{\rho} \mathcal{S}_{\ell}^{+}, g \in C_{c}^{\infty}(0, \infty)\right\} \text {, }
$$


which is a subspace of $C_{c}^{\infty}\left(\mathbf{R}^{3} \backslash\{0\}\right) \otimes \mathbf{C}^{2}$. Note $C_{c}^{\infty}\left(\mathbf{R}^{3} \backslash\{0\}\right) \otimes \mathbf{C}^{2}$ is invariant under the operators $\sigma_{\rho}, \Lambda$ and $L$. By straightforward multiplication, it is easy to verify the following relations:

$$
\sigma \cdot p \sigma_{\rho}=\frac{1}{i} \frac{\partial}{\partial r}-\frac{i}{r}(2+\Lambda), \quad \sigma_{\rho} \sigma \cdot p=\frac{1}{i} \frac{\partial}{\partial r}+\frac{i}{r} \Lambda .
$$

Recall that $\sigma_{\rho}^{2}=1$. Thus if $\xi$ is a unit vector in $\mathcal{S}_{\ell}^{+}$and $f, g \in C_{c}^{\infty}(0, \infty)$, then

$$
\sigma \cdot p \xi f=\sigma_{\rho}\left(\frac{1}{i} \frac{\partial}{\partial r}+\frac{i}{r} \Lambda\right) \xi f=\sigma_{\rho} \xi\left(\frac{1}{i} f^{\prime}+\frac{i}{r} \ell f\right)
$$

and

$$
\sigma \cdot p\left(\sigma_{\rho} \xi g\right)=\left(\frac{1}{i} \frac{\partial}{\partial r}-\frac{i}{r}(2+\Lambda)\right) \xi g=\xi\left(\frac{1}{i} g^{\prime}-\frac{i}{r}(2+\ell) g\right) .
$$

If we define

$$
\Omega_{\xi}=\left\{\xi f+\sigma_{\rho} \xi g: f, g \in C_{c}^{\infty}(0, \infty)\right\}
$$

and

$$
U_{\xi}\left(\xi f+\sigma_{\rho} \xi g\right)=\left[\begin{array}{l}
g \\
f
\end{array}\right],
$$

then $U_{\xi}$ extends to a unitary operator from the closure of $\Omega_{\xi}$ to $L^{2}\left((0, \infty), r^{2} d r\right) \otimes$ $\mathbf{C}^{2}$. Furthermore, $U_{\xi} \Omega_{\xi}=C_{c}^{\infty}(0, \infty) \otimes \mathbf{C}^{2}$ and the above calculation shows that

$$
U_{\xi} \sigma \cdot p=\left[\begin{array}{cc}
0 & -i d / d r+i \ell / r \\
-i d / d r-i(2+\ell) / r & 0
\end{array}\right] U_{\xi} \text { on } \Omega_{\xi} .
$$

Let $W: L^{2}\left((0, \infty), r^{2} d r\right) \otimes \mathbf{C}^{2} \rightarrow L^{2}(0, \infty) \otimes \mathbf{C}^{2}$ be the unitary operator $(W f)(r)$ $=r f(r)$. Then

$$
W\left[\begin{array}{cc}
0 & \frac{1}{i} \frac{d}{d r}+i \frac{\ell}{r} \\
\frac{1}{i} \frac{d}{d r}-i \frac{2+\ell}{r} & 0
\end{array}\right]=\left[\begin{array}{cc}
0 & -i d / d r+i(1+\ell) / r \\
-i d / d r-i(1+\ell) / r & 0
\end{array}\right] W .
$$

Let

$$
J=\frac{1}{2}\left[\begin{array}{cc}
1+i & -1+i \\
1+i & 1-i
\end{array}\right]
$$

We have

$$
J\left[\begin{array}{cc}
0 & -i d / d t+i(1+\ell) / t \\
-i d / d t-i(1+\ell) / t & 0
\end{array}\right]=\left[\begin{array}{cc}
(1+\ell) / t & d / d t \\
-d / d t & -(1+\ell) / t
\end{array}\right] J .
$$

Combining these identities, we obtain

$$
J W U_{\xi}(\sigma \cdot p-Q)=\left(D_{\ell}-Q\right) J W U_{\xi} \text { on } \Omega_{\xi}
$$

for every unit vector $\xi \in \mathcal{S}_{\ell}^{+}$and for every radial function $Q$ which is bounded outside every neighborhood of the origin and measurable.

For each $\ell \in \mathbf{Z}_{+}$, let $\xi_{\ell, 1}, \ldots, \xi_{\ell, 2 \ell+2}$ be an orthonormal basis for $\mathcal{S}_{\ell}^{+}$(with respect to the spherical measure $\sin \gamma d \gamma d \lambda$ ). Then

$$
\Omega_{\ell}=\Omega_{\xi_{\ell, 1}} \oplus \cdots \oplus \Omega_{\xi_{\ell, 2 \ell+2}} .
$$

If we define $U_{\ell}=J W U_{\xi_{\ell, 1}} \oplus \cdots \oplus J W U_{\xi_{\ell, 2 \ell+2}}$, then $U_{\ell}$ extends to a unitary operator from the closure of $\Omega_{\ell}$ in $L^{2}\left(\mathbf{R}^{3}\right) \otimes \mathbf{C}^{2}$ to $\left(L^{2}(0, \infty) \otimes \mathbf{C}^{2}\right)^{[2 \ell+2]}$ and, by (3.4),

$$
U_{\ell}(\sigma \cdot p-Q)=\left(D_{\ell}-Q\right)^{[2 \ell+2]} U_{\ell} \text { on } \Omega_{\ell}
$$


In the above and hereafter, we denote the orthogonal sum of $k$ copies of $X$ by $X^{[k]}$. Also, $U_{\ell} \Omega_{\ell}=\left(C_{c}^{\infty}(0, \infty) \otimes \mathbf{C}^{2}\right)^{[2 \ell+2]}$. Thus we have established the following:

Proposition 3.3. Let $U=U_{0} \oplus U_{1} \oplus \cdots \oplus U_{\ell} \oplus \ldots$ and let $\Omega$ be the algebraic sum $\Omega_{0}+\Omega_{1}+\cdots+\Omega_{\ell}+\ldots$. Then $U$ is a unitary operator from $L^{2}\left(\mathbf{R}^{3}\right) \otimes \mathbf{C}^{2}$ to

$$
\left(L^{2}(0, \infty) \otimes \mathbf{C}^{2}\right)^{[2]} \oplus\left(L^{2}(0, \infty) \otimes \mathbf{C}^{2}\right)^{[4]} \oplus \cdots \oplus\left(L^{2}(0, \infty) \otimes \mathbf{C}^{2}\right)^{[2 \ell+2]} \oplus \ldots .
$$

Moreover,

$$
\begin{aligned}
U \Omega=\left(C_{c}^{\infty}(0, \infty) \otimes \mathbf{C}^{2}\right)^{[2]} \oplus\left(C_{c}^{\infty}(0, \infty) \otimes \mathbf{C}^{2}\right)^{[4]} \oplus \ldots \\
\oplus\left(C_{c}^{\infty}(0, \infty) \otimes \mathbf{C}^{2}\right)^{[2 \ell+2]} \oplus \ldots
\end{aligned}
$$

and, for every radial function $Q$ which is bounded outside every neighborhood of the origin and measurable,

$$
U(\sigma \cdot p-Q)=\left\{\bigoplus_{\ell=0}^{\infty}\left(D_{\ell}-Q\right)^{[2 \ell+2]}\right\} U \text { on } \Omega .
$$

Lemma 3.4. Let $Q$ be a real-valued, measurable, radial function on $\mathbf{R}^{3}$. Suppose that there is a positive number $M$ such that $|Q(r)| \leq M / r$ a.e. on $\mathbf{R}^{3}$. Then the closure of the symmetric operator $\sigma \cdot p-Q$ on $\Omega$ is the same as its closure on $C_{c}^{\infty}\left(\mathbf{R}^{3} \backslash\{0\}\right)$.

Proof. For each $\ell \in \mathbf{Z}_{+}$, the orthogonal projection from $L^{2}\left(\mathbf{R}^{3}\right) \otimes \mathbf{C}^{2}$ onto the closure of $\Omega_{\ell}$ is given by the formula

$$
\left(P_{\ell} f\right)(r, \gamma, \lambda)=\sum_{j=1}^{2 \ell+2} \xi_{\ell, j}(\gamma, \lambda) \int_{0}^{2 \pi} \int_{0}^{\pi} \sin \gamma^{\prime}\left\langle f\left(r, \gamma^{\prime}, \lambda^{\prime}\right), \xi_{\ell, j}\left(\gamma^{\prime}, \lambda^{\prime}\right)\right\rangle_{\mathbf{C}^{2}} d \gamma^{\prime} d \lambda^{\prime}
$$

Each $P_{\ell}$ clearly maps $C_{c}^{\infty}\left(\mathbf{R}^{3} \backslash\{0\}\right) \otimes \mathbf{C}^{2}$ to $\Omega_{\ell}$. Furthermore, if $\varphi \in C_{c}^{\infty}\left(\mathbf{R}^{3} \backslash\{0\}\right)$ vanishes on $\left\{x \in \mathbf{R}^{3}:|x| \leq \delta\right\}$, then so does every $P_{\ell} \varphi$. This implies

$$
\lim _{n \rightarrow \infty}\left\|Q \sum_{\ell=0}^{n} P_{\ell} \varphi-Q \varphi\right\|=0 .
$$

Hence it suffices to show that $\lim _{n \rightarrow \infty}\left\|\sigma \cdot p\left(\sum_{\ell=0}^{n} P_{\ell} \varphi-\varphi\right)\right\|=0$ for such a $\varphi$. But this will become obvious once we show that $\sigma \cdot p P_{\ell} \varphi=P_{\ell} \sigma \cdot p \varphi$.

Because $\Omega_{\ell}$ is invariant under $\sigma \cdot p$, for every $\psi \in \Omega_{\ell}$, we have

$$
\left\langle P_{\ell} \sigma \cdot p \varphi, \psi\right\rangle=\langle\sigma \cdot p \varphi, \psi\rangle=\langle\varphi, \sigma \cdot p \psi\rangle=\left\langle P_{\ell} \varphi, \sigma \cdot p \psi\right\rangle=\left\langle\sigma \cdot p P_{\ell} \varphi, \psi\right\rangle .
$$

Also, if $\psi^{\prime} \in \Omega_{\ell^{\prime}}$ and $\ell^{\prime} \neq \ell$, then $\left\langle P_{\ell} \sigma \cdot p \varphi, \psi^{\prime}\right\rangle=0=\left\langle\sigma \cdot p P_{\ell} \varphi, \psi^{\prime}\right\rangle$. Hence $P_{\ell} \sigma \cdot p \varphi=\sigma \cdot p P_{\ell} \varphi$. This completes the proof.

\section{The FUll Dirac operator}

We will use the following set of Dirac matrices $\alpha_{1}, \alpha_{2}, \alpha_{3}, \alpha_{4}=\beta$ :

$$
\alpha_{j}=\sigma_{j} \otimes \sigma_{3}=\sigma_{j} \oplus\left(-\sigma_{j}\right), \quad j=1,2,3, \quad \text { and } \beta=I_{2} \otimes \sigma_{1}=\left[\begin{array}{cc}
0 & I_{2} \\
I_{2} & 0
\end{array}\right] .
$$

Here $I_{2}$ denotes the $2 \times 2$ identity matrix. This differs from the more commonly used set $\left(\sigma_{1} \otimes \sigma_{1}, \sigma_{2} \otimes \sigma_{1}, \sigma_{3} \otimes \sigma_{1}, I_{2} \otimes \sigma_{3}\right)$. However we feel it is more convenient 
to work with (4.1). Note that the conjugation by the self-adjoint unitary matrix

$$
\frac{1}{\sqrt{2}}\left[\begin{array}{cc}
1 & 1 \\
1 & -1
\end{array}\right]
$$

interchanges $\sigma_{1}$ and $\sigma_{3}$. Therefore our Dirac operator will be the usual one conjugated by $I_{2}$ tensored by the above matrix.

For $\mu_{0} \in \mathbf{R}$, the initial domain of the free Dirac operator

$$
\alpha \cdot p+\mu_{0} \beta=\alpha_{1} p_{1}+\alpha_{2} p_{2}+\alpha_{3} p_{3}+\mu_{0} \beta=(\sigma \cdot p) \oplus(-\sigma \cdot p)+\mu_{0} \beta
$$

is $C_{c}^{\infty}\left(\mathbf{R}^{3}\right) \otimes \mathbf{C}^{4}$. It is well known that this operator is essentially self-adjoint. By Lemmas 3.2 and 3.4, it is even essentially self-adjoint on $C_{c}^{\infty}\left(\mathbf{R}^{3} \backslash\{0\}\right) \otimes \mathbf{C}^{4}$ and on $\Omega \oplus \Omega$.

Theorem 4.1. Suppose that $Q$ is a real-valued, measurable, radial function on $\mathbf{R}^{3}$ and that $|Q(r)| \leq\left(\ell+\frac{1}{2}\right) / r$ a.e. on $\mathbf{R}^{3}$ for some $\ell \in \mathbf{Z}_{+}$. Then the deficiency indices of $\alpha \cdot p+\mu_{0} \beta-Q$ on $C_{c}^{\infty}\left(\mathbf{R}^{3}\right) \otimes \mathbf{C}^{4}$ are $(n, n)$ where

$$
n \leq 2 \ell(\ell+1) \text {. }
$$

Proof. Since the term $\mu_{0} \beta$ is bounded, it suffices to compute the deficiency indices of $\alpha \cdot p-Q=(\sigma \cdot p-Q) \oplus(-\sigma \cdot p-Q)$. Hence we only need to consider the deficiency indices of $\sigma \cdot p \pm Q$ on $C_{c}^{\infty}\left(\mathbf{R}^{3}\right) \otimes \mathbf{C}^{2}$. According to Lemmas 3.2 and 3.4 , for this purpose we may reduce the domain to $\Omega$. By Proposition 3.3, the deficiency indices of $\sigma \cdot p \pm Q$ on $\Omega$ are the same as those of $\bigoplus_{k=0}^{\infty}\left(D_{k} \pm Q\right)^{[2 k+2]}$ on $\bigoplus_{k=0}^{\infty}\left(C_{c}^{\infty}(0, \infty) \otimes \mathbf{C}^{2}\right)^{[2 k+2]}$.

If $\ell=0$, then it follows from Proposition 2.1 that $D_{k} \pm Q$ are essentially selfadjoint on $C_{c}^{\infty}(0, \infty) \otimes \mathbf{C}^{2}$ for all $k \in \mathbf{Z}_{+}$. Hence so is $\alpha \cdot p+\mu_{0} \beta-Q$.

When $\ell \geq 1$, it follows from the same proposition that if $k \geq \ell$, then $D_{k} \pm Q$ are essentially self-adjoint. Hence the deficiency indices of $\sigma \cdot p \pm Q$ coincide with those of $\bigoplus_{k=0}^{\ell-1}\left(D_{k} \pm Q\right)^{[2 k+2]}$ on $\bigoplus_{k=0}^{\ell-1}\left(C_{c}^{\infty}(0, \infty) \otimes \mathbf{C}^{2}\right)^{[2 k+2]}$. It easily follows from the boundedness of $Q$ near infinity that the deficiency indices of $D_{k} \pm Q$ are either $(1,1)$ or $(0,0)$. This completes the proof.

Theorem 4.2. Let $v$ be a real-valued, bounded, measurable function on $(0, \infty)$ such that $v=0$ on $(d, \infty)$ for some $d>0$. Suppose that the limit

$$
v\left(0^{+}\right)=\lim _{\epsilon \downarrow 0} v(\epsilon)
$$

exists. Let $V(r)=v(r) / r$ on $\mathbf{R}^{3} \backslash\{0\}$. $\mathbf{C}^{4}$.

(a) If $\left|v\left(0^{+}\right)\right|<\sqrt{3} / 2$, then $\alpha \cdot p+\mu_{0} \beta-V$ is essentially self-adjoint on $C_{c}^{\infty}\left(\mathbf{R}^{3}\right) \otimes$

(b) If $\sqrt{\ell^{2}-(1 / 4)}<\left|v\left(0^{+}\right)\right|<\sqrt{(1+\ell)^{2}-(1 / 4)}$, $\ell \in \mathbf{N}$, then the deficiency indices of $\alpha \cdot p+\mu_{0} \beta-V$ on $C_{c}^{\infty}\left(\mathbf{R}^{3}\right) \otimes \mathbf{C}^{4}$ are $(2 \ell(\ell+1), 2 \ell(\ell+1))$.

Proof. As in the previous proof, the deficiency indices of $\alpha \cdot p+\mu_{0} \beta-V$ are the sums of those of $\sigma \cdot p \pm V$ on $\Omega$. In the case (a), it follows from Theorem 2.2 that $D_{k} \pm V$ are essentially self-adjoint for every $k \in \mathbf{Z}_{+}$. Hence it follows from Proposition 3.3 and Lemmas 3.2 and 3.4 that $\alpha \cdot p+\mu_{0} \beta-V$ is essentially self-adjoint.

In the case (b), it follows from Theorem 2.2 that the deficiency indices of $D_{k} \pm V$ are $(1,1)$ if $0 \leq k \leq \ell-1$ and $(0,0)$ if $k \geq \ell$. Hence the deficiency indices of $\sigma \cdot p \pm V$ coincide with those of $\bigoplus_{k=0}^{\ell-1}\left(D_{k} \pm V\right)^{[2 k+2]}$, namely $(\ell(\ell+1), \ell(\ell+1))$. 
Lemma 4.3. Let $Q$ be a boundedly supported measurable function on $\mathbf{R}^{3}$ for which there is an $M>0$ such that $|Q(r)| \leq M / r$ a.e. on $\mathbf{R}^{3}$. Then for any $z_{1}, z_{2} \in \mathbf{C} \backslash \mathbf{R}$,

$$
\left(\sigma \cdot p-z_{1}\right)^{-1} Q\left(\sigma \cdot p-z_{2}\right)^{-1}
$$

is a Hilbert-Schmidt operator on $L^{2}\left(\mathbf{R}^{3}\right) \otimes \mathbf{C}^{2}$.

Proof. Since $(\sigma \cdot p-z)(\sigma \cdot p-w)^{-1}$ is bounded for any $z, w \in \mathbf{C} \backslash \mathbf{R}$, it suffices to consider the case where $z_{1}=i$ and $z_{2}=-i$. By the polar decomposition $Q=(Q /|Q|) \times|Q|$ on its support, it suffices to show that if $A$ is a bounded, non-negative self-adjoint operator, then $(\sigma \cdot p-i)^{-1}|Q|^{1 / 2} A|Q|^{1 / 2}(\sigma \cdot p+i)^{-1}$ is a Hilbert-Schmidt operator. If we let $B=A^{1 / 2}|Q|^{1 / 2}(\sigma \cdot p+i)^{-1}$, then

$$
B B^{*}=A^{1 / 2}|Q|^{1 / 2}\left((\sigma \cdot p)^{2}+1\right)^{-1}|Q|^{1 / 2} A^{1 / 2} .
$$

It is well known that

$$
\left((\sigma \cdot p)^{2}+1\right)^{-1}=(-\Delta+1)^{-1}
$$

has kernel function $e^{-|x-y|} / 4 \pi|x-y|$ on $\mathbf{R}^{3} \times \mathbf{R}^{3}$. Straightforward integration shows that $|Q|^{1 / 2}\left((\sigma \cdot p)^{2}+1\right)^{-1}|Q|^{1 / 2}$ is a Hilbert-Schmidt operator. Therefore so are $B B^{*}$ and $B^{*} B=(\sigma \cdot p-i)^{-1}|Q|^{1 / 2} A|Q|^{1 / 2}(\sigma \cdot p+i)^{-1}$.

Theorem 4.4. Let $v$ be a bounded, real-valued measurable function on $(0, \infty)$ which vanishes on $(d, \infty)$ for some $d>0$. Suppose that the limit

$$
\lim _{\epsilon \downarrow 0} v(\epsilon)=v\left(0^{+}\right)
$$

exists. Let $V(r)=v(r) / r$ on $\mathbf{R}^{3}$. Given a real number $\mu_{0}$, let $A_{0}$ denote the closure of $\alpha \cdot p+\mu_{0} \beta$ on $C_{c}^{\infty}\left(\mathbf{R}^{3}\right) \otimes \mathbf{C}^{4}$ and let $A$ be any self-adjoint extension of the symmetric operator $\alpha \cdot p+\mu_{0} \beta-V$ on $C_{c}^{\infty}\left(\mathbf{R}^{3}\right) \otimes \mathbf{C}^{4}$. Then for any $z \in \mathbf{C} \backslash \mathbf{R}$ and any bounded self-adjoint operator $B$ on $L^{2}\left(\mathbf{R}^{3}\right) \otimes \mathbf{C}^{4}$,

$$
(A+B-z)^{-1}-\left(A_{0}+B-z\right)^{-1}
$$

is a Hilbert-Schmidt operator.

Proof. By Lemma 1.1, we only need to consider the case where $\mu_{0}=0$ and $B=0$. Moreover, since the deficiency indices of $\alpha \cdot p-V$ are finite (Theorem 4.1), it suffices to treat a particular self-adjoint extension $A$ of our choosing. Furthermore, because $\alpha \cdot p-V=(\sigma \cdot p-V) \oplus(-\sigma \cdot p-V)$ and because of Lemmas 3.2 and 3.4, it suffices to show that there is a self-adjoint extension $\tilde{A}$ of the symmetric operator $\sigma \cdot p-V$ on $\Omega$ such that $(\tilde{A}-z)^{-1}-(\sigma \cdot p-z)^{-1}$ is a Hilbert-Schmidt operator.

For each $k \in \mathbf{Z}_{+}$, let $T_{k}$ be a self-adjoint extension of $D_{k}-V$. It follows from (3.5) that

$$
\tilde{A}=U^{*}\left\{\bigoplus_{k=0}^{\infty} T_{k}^{[2 k+2]}\right\} U
$$

is a self-adjoint extension of $\sigma \cdot p-V$. Let $\ell \in \mathbf{N}$ be such that $\ell+(1 / 2)>\|v\|_{\infty}$. Let $\Sigma_{1}=\Omega_{0}+\cdots+\Omega_{\ell-1}$ and $\Sigma_{2}=\Omega_{\ell}+\cdots+\Omega_{\ell+n}+\ldots$. Let $\mathcal{H}_{j}$ be the closure of $\Sigma_{j}$ and let $P_{j}: L^{2}\left(\mathbf{R}^{3}\right) \otimes \mathbf{C}^{2} \rightarrow \mathcal{H}_{j}$ be the orthogonal projection, $j=1,2$. The theorem will follow once we show that, for $j=1,2$,

$$
\left((\tilde{A}-z)^{-1}-(\sigma \cdot p-z)^{-1}\right) P_{j}
$$

is a Hilbert-Schmidt operator whenever $z \in \mathbf{C} \backslash \mathbf{R}$. 
Let us first consider the case $j=1$. By (3.5), if $\varphi \in(\sigma \cdot p-z) \Sigma_{1}$, then

$$
\left((\tilde{A}-z)^{-1}-(\sigma \cdot p-z)^{-1}\right) \varphi=U^{*} \bigoplus_{k=0}^{\ell-1}\left\{\left(T_{k}-z\right)^{-1}-\left(D_{k}-z\right)^{-1}\right\}^{[2 k+2]} U \varphi .
$$

It is easy to see that $(\sigma \cdot p-z) \Sigma_{1}$ is dense in $\mathcal{H}_{1}$. Therefore it follows from Theorem 1.7 that $\left((\tilde{A}-z)^{-1}-(\sigma \cdot p-z)^{-1}\right) P_{1}$ is a Hilbert-Schmidt operator.

Now the case $j=2$. It was shown in the proof of Proposition 2.1 that

$$
\left\|t^{-1} \psi\right\| \leq\left\|\left(D_{k}+i\right) \psi\right\| /(k+(1 / 2))
$$

whenever $\psi \in C_{c}^{\infty}(0, \infty) \otimes \mathbf{C}^{2}$. Since $D_{k}$ is essentially self-adjoint on $C_{c}^{\infty}(0, \infty) \otimes \mathbf{C}^{2}$, it follows that

$$
\left\|t^{-1}\left(D_{k}+i\right)^{-1}\right\| \leq 1 /(k+(1 / 2)) .
$$

Therefore when $k \geq \ell$,

$$
\left\|V\left(D_{k}+i\right)^{-1}\right\| \leq\|v\|_{\infty}\left\|t^{-1}\left(D_{k}+i\right)^{-1}\right\| \leq \frac{\|v\|_{\infty}}{k+\frac{1}{2}} \leq \frac{\|v\|_{\infty}}{\ell+\frac{1}{2}}<1 .
$$

If $\eta$ is a bounded radial function, then

$$
U \eta(\sigma \cdot p+i)^{-1}\left|\mathcal{H}_{2}=\bigoplus_{k=\ell}^{\infty}\left\{\eta\left(D_{k}+i\right)^{-1}\right\}^{[2 k+2]} U\right| \mathcal{H}_{2}
$$

By a standard argument we may replace $\eta$ by $V$. Hence

$$
\left\|V(\sigma \cdot p+i)^{-1} P_{2}\right\| \leq \frac{\|v\|_{\infty}}{\ell+\frac{1}{2}}<1 .
$$

Furthermore, $P_{2}$ commutes with $V(\sigma \cdot p+i)^{-1}$, which is a bounded operator because $(\sigma \cdot p)^{2}=-\Delta$. Thus we have the operator

$$
\begin{aligned}
R & =\lim _{N \rightarrow \infty}(\sigma \cdot p+i)^{-1} \sum_{n=0}^{N}\left(V(\sigma \cdot p+i)^{-1}\right)^{n} P_{2} \\
& =(\sigma \cdot p+i)^{-1} P_{2} \sum_{n=0}^{\infty}\left(V(\sigma \cdot p+i)^{-1} P_{2}\right)^{n},
\end{aligned}
$$

where the infinite series converges in the operator norm topology. It is clear that

$$
R(\sigma \cdot p-V+i) \varphi=\varphi \text { whenever } \varphi \in \Sigma_{2} .
$$

Since $(\ell+1)^{2}-\left|v\left(0^{+}\right)\right|^{2} \geq 3 / 4>1 / 4$, it follows from Theorem 2.2 that $D_{k}-V$ is self-adjoint whenever $k \geq \ell$. Since

$$
U(\sigma \cdot p-V)\left|\Sigma_{2}=\left\{\bigoplus_{k=\ell}^{\infty}\left(D_{k}-V\right)^{[2 k+2]}\right\} U\right| \Sigma_{2},
$$

$(\sigma \cdot p-V+i) \Sigma_{2}$ is dense in $\mathcal{H}_{2}$. Hence

$$
(\tilde{A}+i)^{-1} P_{2}=R=(\sigma \cdot p+i)^{-1} P_{2} \sum_{n=0}^{\infty}\left(V(\sigma \cdot p+i)^{-1} P_{2}\right)^{n} .
$$


Lemma 4.3 tells us that $(\sigma \cdot p+i)^{-1} V(\sigma \cdot p+i)^{-1}$ is a Hilbert-Schmidt operator. By $(4.2), \sum_{n=0}^{\infty}\left(V(\sigma \cdot p+i)^{-1} P_{2}\right)^{n}$ is a bounded operator. Therefore

$$
\begin{aligned}
& \left((\tilde{A}+i)^{-1}-(\sigma \cdot p+i)^{-1}\right) P_{2} \\
& \quad=(\sigma \cdot p+i)^{-1} V(\sigma \cdot p+i)^{-1} P_{2} \sum_{n=0}^{\infty}\left(V(\sigma \cdot p+i)^{-1} P_{2}\right)^{n}
\end{aligned}
$$

is a Hilbert-Schmidt operator. This completes the proof.

\section{External ELECTROMAGNETiC FIELDS}

Having treated the self-adjoint extensions of $\alpha \cdot p+\mu_{0} \beta-V$, we will show how to realize $\alpha \cdot p+\mu_{0} \beta-V-V_{1}-\mathcal{E}$ as a self-adjoint operator in $L^{2}\left(\mathbf{R}^{3}\right) \otimes \mathbf{C}^{4}$. Here, $V$ represents the Coulomb singularity as before, $V_{1}$ is a bounded potential, and $\mathcal{E}$ represents an external field. More precisely, the following are fixed throughout this section:

(i) $V(r)=v(r) / r$, where $v$ is a real-valued, bounded, measurable function on $(0, \infty)$ such that $v=0$ on $(d, \infty)$ for some $d>0$ and such that the limit $v\left(0^{+}\right)=$ $\lim _{\epsilon \downarrow 0} v(\epsilon)$ exists. $\mathbf{R}^{3}$.

(ii) $V_{1}$ is a $4 \times 4$ self-adjoint matrix-valued, bounded, measurable function on

(iii) $\mathcal{E}$ is a $4 \times 4$ self-adjoint matrix-valued, measurable function on $\mathbf{R}^{3}$ which is bounded on every bounded subset of $\mathbf{R}^{3}$ and which can be unbounded at infinity.

(iv) For each $n \in \mathbf{N}, \mathcal{E}_{n}$ is defined to be the function such that $\mathcal{E}_{n}(x)=\mathcal{E}(x)$ when $|x| \leq n$ and $\mathcal{E}_{n}(x)=0$ when $|x|>n$.

It is known from Chernoff's work that the addition of a potential which is locally bounded but which may blow up at infinity to the Dirac operator does not alter its essential self-adjointness. See [2, Theorem 2.1]. But we will go one step further. We will show that, with the addition of such a potential, removing the Coulomb singularity at 0 still only results in a Hilbert-Schmidt perturbation of the resolvent of the Dirac operator.

Let $\mathcal{R}$ denote the collection of $C^{\infty}$-functions $0 \leq \eta \leq 1$ on $[0, \infty)$ for which there exist $0<d(\eta)<M(\eta)<\infty$ such that $\eta=1$ on $[0, d(\eta)]$ and $\eta=0$ on $[M(\eta), \infty)$. Each $\eta \in \mathcal{R}$ will also be identified with the radial function $x \mapsto \eta(|x|)$ on $\mathbf{R}^{3}$. As such, each $\eta \in \mathcal{R}$ is a compactly supported $C^{\infty}$-function on $\mathbf{R}^{3}$. Let

$$
\alpha_{\rho}=\sigma_{\rho} \oplus\left(-\sigma_{\rho}\right) .
$$

Proposition 5.1. Let $A$ be a self-adjoint extension of the minimal symmetric operator $\alpha \cdot p+\mu_{0} \beta-V-V_{1}$ on $C_{c}^{\infty}\left(\mathbf{R}^{3}\right) \otimes \mathbf{C}^{4}$ and let $\mathcal{D}(A)$ denote the domain of A. Then the following hold true:

(a) For each $f \in \mathcal{R}, f \mathcal{D}(A) \subset \mathcal{D}(A)$.

(b) $\mathcal{D}_{c}(A)=\{u \in \mathcal{D}(A):$ u has a bounded support $\}$ is a core for $A$.

(c) For each $f \in \mathcal{R},[A, f]$ is the operator of multiplication by $-i \sum_{j=1}^{3} \alpha_{j} \partial f / \partial x_{j}$ $=-i \alpha_{\rho} f^{\prime}$.

Proof. Because $V_{1}$ is bounded, $A$ is a self-adjoint extension of $\alpha \cdot p-V$ plus $\mu_{0} \beta-V_{1}$. Furthermore, since $\alpha \cdot p-V=(\sigma \cdot p-V) \oplus(-\sigma \cdot p-V)$ and since there is no restriction on the sign of $V$, it suffices to show that if $S$ is a self-adjoint extension of $\sigma \cdot p-V$ with domain $\mathcal{D}(S) \subset L^{2}\left(\mathbf{R}^{3}\right) \otimes \mathbf{C}^{2}$, then $f \mathcal{D}(S) \subset \mathcal{D}(S)$ for every $f \in \mathcal{R}$, 
that $\mathcal{D}_{c}(S)=\{u \in \mathcal{D}(S): u$ has a bounded support $\}$ is a core for $S$, and that $[S, f]=-i \sigma_{\rho} f^{\prime}$.

If $U$ is the unitary operator in Proposition 3.3, then $U S U^{*}$ is a self-adjoint extension of the symmetric operator $\bigoplus_{k=0}^{\infty}\left(D_{k}-V\right)^{[2 k+2]}$ on the algebraic sum $\bigoplus_{k=0}^{\infty}\left[C_{c}^{\infty}(0, \infty) \otimes \mathbf{C}^{2}\right]^{[2 k+2]}=U \Omega$. Let $\ell \in \mathbf{N}$ be such that $\ell \geq\|v\|_{\infty}$. It follows from Proposition 2.1 that $D_{k}-V$ is self-adjoint whenever $k \geq \ell$. Thus $U S U^{*}=$ $S_{1} \oplus S_{2}$, where $S_{1}$ is a self-adjoint extension of $\bigoplus_{k=0}^{\ell-1}\left(D_{k}-V\right)^{[2 k+2]}$ and $S_{2}$ is the closure of $\bigoplus_{k=\ell}^{\infty}\left(D_{k}-V\right)^{[2 k+2]}$. Let $\mathcal{D}\left(S_{j}\right)$ denote the domain of $S_{j}, j=1,2$.

Since $f$ is a radial function, we have $U M_{f}^{3} U^{*}=\bigoplus_{k=0}^{\infty}\left(M_{f}^{1}\right)^{[2 k+2]}$, where $M^{n}$ denotes the multiplication on $\mathbf{R}^{n}$ or subsets thereof. Since $f C_{c}^{\infty}(0, \infty) \otimes \mathbf{C}^{2} \subset$ $C_{c}^{\infty}(0, \infty) \otimes \mathbf{C}^{2}$, we have $f \mathcal{D}\left(S_{2}\right) \subset \mathcal{D}\left(S_{2}\right)$. On the other hand, for each $k \leq$ $\ell-1$, the orthogonal complement $N_{k}(\mp i)$ of $\left(D_{k}-V \pm i\right) C_{c}^{\infty}(0, \infty) \otimes \mathbf{C}^{2}$ consists of the solutions of the differential equation $\left(D_{k}-V \mp i\right) u=0$ which belong to $L^{2}(0, \infty) \otimes \mathbf{C}^{2}$. Elementary theory of differential equations tells us that any $\varphi \in$ $\left\{\bigoplus_{k=0}^{\ell-1}\left(N_{k}(-i)\right)^{[2 k+2]}\right\}+\left\{\bigoplus_{k=0}^{\ell-1}\left(N_{k}(i)\right)^{[2 k+2]}\right\}$ must be absolutely continuous on any compact interval in $(0, \infty)$. Thus $(1-f) \varphi$ belongs to the domain of the closure of $\bigoplus_{k=0}^{\ell-1}\left(D_{k}-V\right)^{[2 k+2]}$ on $\bigoplus_{k=0}^{\ell-1}\left(C_{c}^{\infty}(0, \infty) \otimes \mathbf{C}^{2}\right)^{[2 k+2]}$. von Neumann's formula for self-adjoint extensions now tells us that $(1-f) \mathcal{D}\left(S_{1}\right)$ is contained in the same domain. Hence $f \mathcal{D}\left(S_{1}\right) \subset \mathcal{D}\left(S_{1}\right)$. Applying the inverse unitary transformation, we obtain $f \mathcal{D}(S) \subset \mathcal{D}(S)$.

It also follows from von Neumann's formula for self-adjoint extensions that for any $\psi \in \mathcal{D}\left(S_{1}\right), S_{1} \psi$ is obtained from $\psi$ by the direct application of the differential expression $\bigoplus_{k=0}^{\ell-1}\left(D_{k}-V\right)^{[2 k+2]}$ on $(0, \infty)$. Hence straightforward differentiation yields

$$
S_{1} f \varphi=f S_{1} \varphi+i\left\{\bigoplus_{k=0}^{\ell-1} \sigma_{2}^{[2 k+2]}\right\} f^{\prime} \varphi .
$$

for $\varphi \in \mathcal{D}\left(S_{1}\right)$. Similarly,

$$
S_{2} f \psi=f S_{2} \psi+i\left\{\bigoplus_{k=\ell}^{\infty} \sigma_{2}^{[2 k+2]}\right\} f^{\prime} \psi
$$

if $\psi \in \mathcal{D}\left(S_{2}\right)$. Thus $[S, f]=U^{*}\left[S_{1} \oplus S_{2}, f\right] U=i f^{\prime} U^{*}\left\{\bigoplus_{k=0}^{\infty} \sigma_{2}^{[2 k+2]}\right\} U$. Hence it only remains to be shown that $-\bigoplus_{k=0}^{\infty} \sigma_{2}^{[2 k+2]}=U \sigma_{\rho} U^{*}$.

For this purpose we need the unitary operators $U_{\xi}, W, J$ defined in Section 3. $\sigma_{\rho}$ preserves each $\Omega_{\xi}$ and $U_{\xi} \sigma_{\rho}=\sigma_{1} U_{\xi}$ on $\Omega_{\xi}$. The operator $(W g)(r)=r g(r)$ from $L^{2}((0, \infty), r d r) \otimes \mathbf{C}^{2}$ to $L^{2}(0, \infty) \otimes \mathbf{C}^{2}$ commutes with $\sigma_{1}$. Finally, $J \sigma_{1}=-\sigma_{2} J$. That is, $J W U_{\xi} \sigma_{\rho}=-\sigma_{2} J W U_{\xi}$ on $\Omega_{\xi}$.

It follows from the above analysis that for any $f \in \mathcal{R}$,

$$
\left\|\left[S, f_{n}\right]\right\|=\left\|f^{\prime}\right\|_{\infty} / n \rightarrow 0
$$

if $f_{n}(r)=f(r / n)$. This immediately implies that $\mathcal{D}_{c}(S)$ is a core for $S$.

Theorem 5.2. Let $A$ be a self-adjoint extension of the minimal symmetric operator $\alpha \cdot p+\mu_{0} \beta-V-V_{1}$ on $C_{c}^{\infty}\left(\mathbf{R}^{3}\right) \otimes \mathbf{C}^{4}$ and let $\mathcal{D}(A)$ denote the domain of $A$. Let

$$
\mathcal{D}_{c}(A)=\{u \in \mathcal{D}(A): u \text { has a bounded support }\} \text {. }
$$


Then the closure $B$ of the symmetric operator $A-\mathcal{E}$ on $\mathcal{D}_{c}(A)$ is a self-adjoint operator. Furthemore,

$$
\lim _{n \rightarrow \infty}\left(A-\mathcal{E}_{n}-z\right)^{-1}=(B-z)^{-1}
$$

in the strong operator topology for every $z \in \mathbf{C} \backslash \mathbf{R}$.

Proof. Let $z \in \mathbf{C} \backslash \mathbf{R}$. To show that $A-\mathcal{E}$ is essentially self-adjoint on $\mathcal{D}_{c}(A)$, it suffices to show that if $\varphi \in L^{2}\left(\mathbf{R}^{3}\right) \otimes \mathbf{C}^{4}$ is orthogonal to $(A-\mathcal{E}-z) \mathcal{D}_{c}(A)$, then $\varphi=0$. Let $f \in \mathcal{R}$ be such that $f=1$ on $[0,1 / 2]$ and $f=0$ on $[1, \infty)$. Define $f_{n}(r)=$ $f(r / n)$. Then $\mathcal{E}_{n} f_{n}=\mathcal{E} f_{n}$. Now $\left(A-\mathcal{E}_{n}-z\right)^{-1} \varphi$ belongs to the domain of $A-\mathcal{E}_{n}$, which is the same as $\mathcal{D}(A)$. Thus $f_{n}\left(A-\mathcal{E}_{n}-z\right)^{-1} \varphi \in \mathcal{D}_{c}(A)$ according Proposition 5.1(a) and $\varphi \perp(A-\mathcal{E}-z) f_{n}\left(A-\mathcal{E}_{n}-z\right)^{-1} \varphi=\left(A-\mathcal{E}_{n}-z\right) f_{n}\left(A-\mathcal{E}_{n}-z\right)^{-1} \varphi$. Therefore

$$
\begin{aligned}
\|\varphi\|^{2}=\lim _{n \rightarrow \infty}\left\langle\varphi, f_{n} \varphi\right\rangle & =\lim _{n \rightarrow \infty}\left\langle\varphi, f_{n}\left(A-\mathcal{E}_{n}-z\right)\left(A-\mathcal{E}_{n}-z\right)^{-1} \varphi\right\rangle \\
& =\lim _{n \rightarrow \infty}\left\langle\varphi,\left[f_{n}, A-\mathcal{E}_{n}-z\right]\left(A-\mathcal{E}_{n}-z\right)^{-1} \varphi\right\rangle \\
& =\lim _{n \rightarrow \infty}\left\langle\varphi,\left[f_{n}, A\right]\left(A-\mathcal{E}_{n}-z\right)^{-1} \varphi\right\rangle .
\end{aligned}
$$

By Proposition 5.1(c),

$$
\left\|\left[f_{n}, A\right]\left(A-\mathcal{E}_{n}-z\right)^{-1} \varphi\right\| \leq\|\varphi\|\left\|f_{n}^{\prime}\right\|_{\infty} /|\operatorname{Im} z|=\|\varphi\|\left\|f^{\prime}\right\|_{\infty} / n|\operatorname{Im} z| .
$$

Therefore $\|\varphi\|^{2}=0$, as was to be proved.

Let $\psi \in \mathcal{D}_{c}(A)$. If $n$ is large enough that $\mathcal{E}_{n} \psi=\mathcal{E} \psi$, then

$$
\begin{aligned}
\left(A-\mathcal{E}_{n}-z\right)^{-1}(A-\mathcal{E}-z) \psi & =\left(A-\mathcal{E}_{n}-z\right)^{-1}\left(A-\mathcal{E}_{n}-z\right) \psi=\psi \\
& =(B-z)^{-1}(A-\mathcal{E}-z) \psi
\end{aligned}
$$

Since $(A-\mathcal{E}-z) \mathcal{D}_{c}(A)$ is dense in $L^{2}\left(\mathbf{R}^{3}\right) \otimes \mathbf{C}^{4},\left(A-\mathcal{E}_{n}-z\right)^{-1} \rightarrow(B-z)^{-1}$ in the strong operator topology.

If we write $A_{0}$ for the closure of $\alpha \cdot p+\mu_{0} \beta-V_{1}$ on $C_{c}^{\infty}\left(\mathbf{R}^{3}\right) \otimes \mathbf{C}^{4}$, then it follows from Theorem 5.2 that the closure $B_{0}$ of $A_{0}-\mathcal{E}$ on $\mathcal{D}_{c}\left(A_{0}\right)=\left\{u \in \mathcal{D}\left(A_{0}\right): u\right.$ has a bounded support $\}$ is a self-adjoint operator. It is quite obvious that $C_{c}^{\infty}\left(\mathbf{R}^{3}\right) \otimes \mathbf{C}^{4}$ is also a core for $B_{0}$.

Theorem 5.3. Let $A$ and $B$ be the same as in Theorem 5.2 and let $B_{0}$ be defined as above. Then for every $z \in \mathbf{C} \backslash \mathbf{R}$,

$$
(B-z)^{-1}-\left(B_{0}-z\right)^{-1}
$$

is a Hilbert-Schmidt operator.

Proof. Let $\eta \in \mathcal{R}$ and suppose $M>0$ is such that $\eta(r)=0$ when $r \geq M$. Let $\zeta \in \mathcal{R}$ be such that $\zeta(r)=1$ when $0 \leq r \leq M$. In particular $\zeta=1$ on the support of $\eta$. Let $n_{0} \in \mathbf{N}$ be such that $\zeta(r)=0$ when $r \geq n_{0}-1$. 
For any $m \geq n_{0}$, we have $\left(\mathcal{E}_{m}-\mathcal{E}_{n_{0}}\right) \zeta=\left(\mathcal{E}_{m}-\mathcal{E}_{n_{0}}\right) \eta=0$. Therefore

$$
\begin{aligned}
\{(A- & \left.\left.\mathcal{E}_{m}-i\right)^{-1}-\left(A-\mathcal{E}_{n_{0}}-i\right)^{-1}\right\} \eta \\
= & \left(A-\mathcal{E}_{m}-i\right)^{-1}\left(\mathcal{E}_{m}-\mathcal{E}_{n_{0}}\right)\left(A-\mathcal{E}_{n_{0}}-i\right)^{-1} \eta \\
= & \left(A-\mathcal{E}_{m}-i\right)^{-1}\left(\mathcal{E}_{m}-\mathcal{E}_{n_{0}}\right)\left[\left(A-\mathcal{E}_{n_{0}}-i\right)^{-1}, \eta\right] \\
= & \left(A-\mathcal{E}_{m}-i\right)^{-1}\left(\mathcal{E}_{m}-\mathcal{E}_{n_{0}}\right)\left(A-\mathcal{E}_{n_{0}}-i\right)^{-1}[\eta, A]\left(A-\mathcal{E}_{n_{0}}-i\right)^{-1} \\
= & \left\{\left(A-\mathcal{E}_{m}-i\right)^{-1}\left(\mathcal{E}_{m}-\mathcal{E}_{n_{0}}\right)\left(A-\mathcal{E}_{n_{0}}-i\right)^{-1} \zeta\right\} \\
& \times\left\{[\eta, A]\left(A-\mathcal{E}_{n_{0}}-i\right)^{-1}\right\} \\
= & \left(A-\mathcal{E}_{m}-i\right)^{-1}\left(\mathcal{E}_{m}-\mathcal{E}_{n_{0}}\right)\left(A-\mathcal{E}_{n_{0}}-i\right)^{-1} \\
& \times[\zeta, A]\left(A-\mathcal{E}_{n_{0}}-i\right)^{-1}[\eta, A]\left(A-\mathcal{E}_{n_{0}}-i\right)^{-1} \\
= & \left\{\left(A-\mathcal{E}_{m}-i\right)^{-1}-\left(A-\mathcal{E}_{n_{0}}-i\right)^{-1}\right\} K,
\end{aligned}
$$

where, by Proposition 5.1,

$$
K=-\alpha_{\rho} \zeta^{\prime}\left(A-\mathcal{E}_{n_{0}}-i\right)^{-1} \alpha_{\rho} \eta^{\prime}\left(A-\mathcal{E}_{n_{0}}-i\right)^{-1} .
$$

Letting $m \rightarrow \infty$, we obtain

$$
\left\{(B-i)^{-1}-\left(A-\mathcal{E}_{n_{0}}-i\right)^{-1}\right\} \eta=\left\{(B-i)^{-1}-\left(A-\mathcal{E}_{n_{0}}-i\right)^{-1}\right\} K .
$$

We claim that $K$ is a Hilbert-Schmidt operator. Indeed if we let

$$
K_{0}=-\alpha_{\rho} \zeta^{\prime}\left(A_{0}-\mathcal{E}_{n_{0}}-i\right)^{-1} \alpha_{\rho} \eta^{\prime}\left(A_{0}-\mathcal{E}_{n_{0}}-i\right)^{-1},
$$

then it follows from Theorem 4.4 that $K-K_{0}$ is a Hilbert-Schmidt operator. Let $\mathcal{C}_{4}$ denote the collection of compact operators $T$ such that $\operatorname{tr}\left(|T|^{4}\right)<\infty$. From the proof of Lemma 4.3 we see that for any $f \in \mathcal{R}, f^{\prime}(\alpha \cdot p-i)^{-1} \in \mathcal{C}_{4}$. Lemma 1.1 tells us, therefore, that $f^{\prime}\left(A_{0}-\mathcal{E}_{n_{0}}-i\right)^{-1} \in \mathcal{C}_{4}$. This implies $K_{0}$ is a Hilbert-Schmidt operator (see [4, p. 92]). Therefore so is $K$.

Thus we have proved that $\left\{(B-i)^{-1}-\left(A-\mathcal{E}_{n_{0}}-i\right)^{-1}\right\} \eta$ is a Hilbert-Schmidt operator. This, of course, holds true in the special case $V=0$. Therefore $\left\{\left(B_{0}-i\right)^{-1}-\left(A_{0}-\mathcal{E}_{n_{0}}-i\right)^{-1}\right\} \eta$ is a Hilbert-Schmidt operator. Theorem 4.4 tells us that $\left(A-\mathcal{E}_{n_{0}}-i\right)^{-1}-\left(A_{0}-\mathcal{E}_{n_{0}}-i\right)^{-1}$ is a Hilbert-Schmidt operator. Combining these facts, we conclude that for any $\eta \in \mathcal{R},\left\{(B-i)^{-1}-\left(B_{0}-i\right)^{-1}\right\} \eta$ is a Hilbert-Schmidt operator.

Recall that $V(r)=v(r) / r$ with $v=0$ on $(d, \infty)$. Let $\eta \in \mathcal{R}$ be such that $\eta(r)=1$ for $0 \leq r \leq d+1$. Let us now prove that for such an $\eta$, the operator $\left\{(B-i)^{-1}-\left(B_{0}-i\right)^{-1}\right\}(1-\eta)$ is also Hilbert-Schmidt. Let $\varphi \in C_{c}^{\infty}\left(\mathbf{R}^{3}\right) \otimes \mathbf{C}^{4}$. Since $(1-\eta) V=0$, we have $(1-\eta)\left(A_{0}-\mathcal{E}_{m}-i\right) \varphi=(1-\eta)\left(A-\mathcal{E}_{m}-i\right) \varphi$. Thus

$$
\begin{aligned}
\left(A-\mathcal{E}_{m}-i\right)^{-1} & (1-\eta)\left(A_{0}-\mathcal{E}_{m}-i\right) \varphi \\
& =\left(A-\mathcal{E}_{m}-i\right)^{-1}\left[1-\eta, A-\mathcal{E}_{m}-i\right] \varphi+(1-\eta) \varphi \\
& =\left(A-\mathcal{E}_{m}-i\right)^{-1}[A, \eta]\left(A_{0}-\mathcal{E}_{m}-i\right)^{-1}\left(A_{0}-\mathcal{E}_{m}-i\right) \varphi+(1-\eta) \varphi .
\end{aligned}
$$

Since $\left(A_{0}-\mathcal{E}_{m}-i\right) C_{c}^{\infty}\left(\mathbf{R}^{3}\right) \otimes \mathbf{C}^{4}$ is dense in $L^{2}\left(\mathbf{R}^{3}\right) \otimes \mathbf{C}^{4}$, the above yields

$$
\begin{aligned}
\left\{\left(A-\mathcal{E}_{m}-i\right)^{-1}-\right. & \left.\left(A_{0}-\mathcal{E}_{m}-i\right)^{-1}\right\}(1-\eta) \\
& =-i\left\{\left(A-\mathcal{E}_{m}-i\right)^{-1}-\left(A_{0}-\mathcal{E}_{m}-i\right)^{-1}\right\} \alpha_{\rho} \eta^{\prime}\left(A_{0}-\mathcal{E}_{m}-i\right)^{-1} .
\end{aligned}
$$

Letting $m \rightarrow \infty$, we obtain

$$
\left\{(B-i)^{-1}-\left(B_{0}-i\right)^{-1}\right\}(1-\eta)=-i\left\{(B-i)^{-1}-\left(B_{0}-i\right)^{-1}\right\} \alpha_{\rho} \eta^{\prime}\left(B_{0}-i\right)^{-1},
$$


which is a Hilbert-Schmidt operator because $\eta^{\prime}$ has a bounded support. This proves that the operator $(B-i)^{-1}-\left(B_{0}-i\right)^{-1}$ is Hilbert-Schmidt. Another application of Lemma 1.1 yields the full theorem.

\section{Bound States and Relativistic Uncertainty}

We will now assume that $\mu_{0} \neq 0$ and consider the bound states in the gap $\left(-\left|\mu_{0}\right|,\left|\mu_{0}\right|\right)$ in the essential spectrum of the self-adjoint extensions of the minimal operator $\alpha \cdot p+\mu_{0} \beta-V-W$, where $V(r)=v(r) / r$ as in the previous sections and $W$ is a bounded, real-valued, radial function which vanishes in a neighborhood of 0 and at infinity.

For each $k \in \mathbf{Z}_{+}$, define the differential operator

$$
\tilde{D}_{k}=\left[\begin{array}{cc}
D_{k} & \mu_{0} \\
\mu_{0} & -D_{k}
\end{array}\right] \text {. }
$$

By Section 3 it is obvious that $\alpha \cdot p+\mu_{0} \beta-V-W$ on $\Omega \oplus \Omega$ is unitarily equivalent to $\bigoplus_{k=0}^{\infty} \tilde{D}_{k}^{[2 k+2]}-V-W$ on $\bigoplus_{k=0}^{\infty}\left(C_{c}^{\infty}(0, \infty) \otimes \mathbf{C}^{4}\right)^{[2 k+2]}$. For $\ell \geq m$ in $\mathbf{Z}_{+}$, let $P_{\ell, m}$ denote the orthogonal projection on $\bigoplus_{k=0}^{\ell}\left(L^{2}(0, \infty) \otimes \mathbf{C}^{4}\right)^{[2 k+2]}=\left\{f_{0} \oplus \cdots \oplus f_{\ell}\right.$ : $\left.f_{k} \in\left(L^{2}(0, \infty) \otimes \mathbf{C}^{4}\right)^{[2 k+2]}, 0 \leq k \leq \ell\right\}$ defined by the formula

$$
P_{\ell, m}\left(f_{0} \oplus \cdots \oplus f_{\ell}\right)=f_{0} \oplus \cdots \oplus f_{m} \oplus 0 \oplus \cdots \oplus 0 .
$$

Recall that $V_{b}(t)=b \chi_{(0, d)}(t) / t$. Given $k \in \mathbf{Z}_{+}, b \in \mathbf{R}$ and $z \in \mathbf{C}$, let $\tilde{\Psi}_{k, b, z}$ denote the fundamental solution matrix for homogeneous system

$$
\left(\tilde{D}_{k}-V_{b}\right) u=z u \text {. }
$$

If $(1+k)^{2}-b^{2}<0$, then $\tilde{\Psi}_{k, b, z}$ is bounded on every finite interval $(0, c)$. This assertion can be proved as follows. Let $\tilde{\Phi}_{b, k}=\Phi_{k, b} \oplus \Phi_{-b, k}$, where $\Phi_{b, k}$ and $\Phi_{-b, k}$ are given by (1.1). If $u=\tilde{\Phi}_{b, k} c$ solves the above system, then $\tilde{B} \tilde{\Phi}_{b, k} c^{\prime}=M\left(z, \mu_{0}\right) \tilde{\Phi}_{b, k} c$, where

$$
M\left(z, \mu_{0}\right)=\left[\begin{array}{cccc}
z & 0 & -\mu_{0} & 0 \\
0 & z & 0 & -\mu_{0} \\
-\mu_{0} & 0 & z & 0 \\
0 & -\mu_{0} & 0 & z
\end{array}\right]
$$

and $\tilde{B}=B \oplus(-B)$ with $B$ being the matrix defined just before Lemma 1.3. Solving the equation $c^{\prime}=\tilde{\Phi}_{b, k}^{-1} \tilde{B}^{-1} M\left(z, \mu_{0}\right) \tilde{\Phi}_{b, k} c$ using the usual "non-commutative exponential" and recalling the fact that $\tilde{\Phi}_{b, k}$ is bounded on any $(0, c)$ when $(1+k)^{2}-b^{2}<0$, we find that $\tilde{\Psi}_{k, b, z}(t)=\tilde{\Phi}_{b, k}(t)[1+O(t)]$ near 0 .

Proposition 6.1. Suppose that $\left|v\left(0^{+}\right)\right|>1+m$ for some $m \in \mathbf{Z}_{+}$and that $\ell \geq m$. Suppose that $v$ satisfies either

$$
\int_{0}^{d} \frac{\left|v(t)-v\left(0^{+}\right)\right|}{t} d t<\infty
$$

or

$$
\int_{0}^{d} \frac{\left|v(t)-v\left(0^{+}\right)\right|^{2}}{t^{2 \nu}} d t<\infty \text { for some } \quad \nu>1 / 2
$$


Let $A$ be a self-adjoint extension in $\bigoplus_{k=0}^{\ell}\left(L^{2}(0, \infty) \otimes \mathbf{C}^{4}\right)^{[2 k+2]}$ of the symmetric operator $\bigoplus_{k=0}^{\ell} \tilde{D}_{k}^{[2 k+2]}-V-W$ on $\bigoplus_{k=0}^{\ell}\left(C_{c}^{\infty}(0, \infty) \otimes \mathbf{C}^{4}\right)^{[2 k+2]}$. Then for any eigenvector $\psi$ of $A$ such that $P_{\ell, m} \psi \neq 0$, we have

$$
\liminf _{n \rightarrow \infty} \int_{2^{-(n+1)}}^{2^{-n}} \frac{1}{t}|\psi(t)|^{2} d t>0
$$

Proof. Suppose that $A \psi=\lambda \psi$ with $\lambda \in \mathbf{R}$. By von Neumann's formula for selfadjoint extensions (see, e.g., $[7$, p. 35]), $\psi$ solves the linear system

$$
\left(\bigoplus_{k=0}^{\ell} \tilde{D}_{k}^{[2 k+2]}-V-W\right) \psi=\lambda \psi
$$

That $W=0$ near 0 means that, when restricted to a suitable interval $\left(0, c_{1}\right), W$ can be dropped from the above equation. Decomposing $\psi$ into components, it suffices to show that if $\xi$ satisfies the differential equation

$$
\left(\tilde{D}_{k}-V\right) \xi=\lambda \xi
$$

on $(0, c)$ for some $0<c \leq d$ and $0 \leq k \leq m$, and if

$$
\liminf _{n \rightarrow \infty} \int_{2^{-(n+1)}}^{2^{-n}} \frac{1}{t}|\xi(t)|^{2} d t=0,
$$

then $\xi$ is identically zero.

Write $b$ for $v\left(0^{+}\right)$and define

$$
(R g)(t)=\tilde{\Psi}_{b, k, \lambda}(t) \int_{0}^{t} \tilde{\Psi}_{b, k, \lambda}^{-1}(s) \tilde{B}^{-1} g(s) d s .
$$

By the discussion preceding the proposition, the condition $\left|v\left(0^{+}\right)\right|>1+m \geq 1+k$ ensures that both $\tilde{\Psi}_{b, k, \lambda}$ and $\tilde{\Psi}_{b, k, \lambda}^{-1}$ are bounded on some $(0, c)$. It follows from the boundedness of the operator $\left(T_{0} f\right)(t)=t^{-1} \int_{0}^{t} f(s) d s$ that there is a $\delta \in(0, c)$ such that $\left\|\left(V-V_{b}\right) \chi_{(0, \delta)} R \chi_{(0, c)}\right\| \leq 1 / 2$. Let

$$
R^{\prime}=R \sum_{p=0}^{\infty}\left(\left(V-V_{b}\right) \chi_{(0, \delta)} R\right)^{p}
$$

Then $\chi_{(0, c)} R^{\prime} \chi_{(0, c)}$ is a bounded operator.

Let $\eta$ be a $C^{\infty}$-function on $[0, \infty)$ such that $\eta=0$ on $[0,1 / 2]$ and $\eta=1$ on $[1, \infty)$. Let $\eta_{n}(t)=\eta\left(2^{n} t\right)$ for each $n \in \mathbf{N}$. Let $\zeta$ be a $C^{\infty}$-function on $[0, \infty)$ such that $\zeta=1$ on $[0, \delta / 2]$ and $\zeta=0$ on $[2 \delta / 3, \infty)$.

By an argument we used in the proof of Lemma 1.5, for every $\varphi \in C_{c}^{\infty}(0, \infty) \otimes \mathbf{C}^{4}$, $\varphi=R\left(\tilde{D}_{k}-V_{b}-\lambda\right) \varphi$. Thus

$$
\varphi=R^{\prime}\left(\tilde{D}_{k}-V_{b}-\left(V-V_{b}\right) \chi_{(0, \delta)}-\lambda\right) \varphi
$$

for such $\varphi$ if its support is contained in $(0, c)$. It follows from a routine approximation argument that $\eta_{n} \zeta \xi=R^{\prime}\left(\tilde{D}_{k}-V_{b}-\left(V-V_{b}\right) \chi_{(0, \delta)}-\lambda\right) \eta_{n} \zeta \xi$. Since $\left(V_{b}+\left(V-V_{b}\right) \chi_{(0, \delta)}\right) \zeta=V \zeta$, we have

$$
\eta_{n} \zeta \xi=R^{\prime}\left(\tilde{D}_{k}-V-\lambda\right) \eta_{n} \zeta \xi
$$


On the other hand, since $\left(\tilde{D}_{k}-V-\lambda\right) \xi=0$ and $\zeta=1$ on $(0, \delta / 2)$, straightforward differentiation yields

$$
\left(\left(\tilde{D}_{k}-V-\lambda\right) \eta_{n} \zeta \xi\right)(t)=\left(\left(\tilde{D}_{k}-V-\lambda\right) \eta_{n} \xi\right)(t)=\eta_{n}^{\prime}(t) \tilde{B} \xi(t)
$$

when $t \in(0, \delta / 2)$. Note that on $(0, a) R f$ is independent of the values of $f$ on $(a, \infty)$. Therefore

$$
\eta_{n}(t) \xi(t)=\left(R^{\prime} \eta_{n}^{\prime} \tilde{B} \xi\right)(t) \text { if } t \in(0, \delta / 2)
$$

Set

$$
C=\left\|\chi_{(0, c)} \tilde{\Psi}_{b, k, \lambda}\right\|_{\infty}\left\|\chi_{(0, c)} \tilde{\Psi}_{b, k, \lambda}^{-1}\right\|_{\infty}
$$

and

$$
J_{n}=\int_{2^{-(n+1)}}^{2^{-n}} \frac{1}{s}|\xi(s)|^{2} d s .
$$

For $t \in(0, \delta / 2)$, we have the estimate

$$
\begin{aligned}
\left|\left(R \eta_{n}^{\prime} \tilde{B} \xi\right)(t)\right| & \leq C \int_{0}^{t}\left|\eta _ { n } ^ { \prime } ( s ) \left\|\xi(s)\left|d s \leq C\left\|\eta^{\prime}\right\|_{\infty} \int_{2^{-(n+1)}}^{2^{-n}} 2^{n}\right| \xi(s) \mid d s\right.\right. \\
& \leq C\left\|\eta^{\prime}\right\|_{\infty}\left[\int_{2^{-(n+1)}}^{2^{-n}} 2^{n}|\xi(s)|^{2} d s \int_{2^{-(n+1)}}^{2^{-n}} 2^{n} d s\right]^{1 / 2} \leq C\left\|\eta^{\prime}\right\|_{\infty} J_{n}^{1 / 2} .
\end{aligned}
$$

Let us now assume that $\left|v(t)-v\left(0^{+}\right)\right| / t$ is integrable near 0 . Then there is a $\sigma \in(0, \delta / 2)$ such that $C \int_{0}^{\sigma} s^{-1}\left|v(s)-v\left(0^{+}\right)\right| d s \leq 1 / 2$. Thus for any $p \geq 1$ and $t \in(0, \sigma)$,

$$
\begin{aligned}
\left|\left(\left(R\left(V-V_{b}\right)\right)^{p} R \eta_{n}^{\prime} \tilde{B} \xi\right)(t)\right| & \leq C^{p}\left[\int_{0}^{t} \frac{\left|v(s)-v\left(0^{+}\right)\right|}{s} d s\right]^{p} C\left\|\eta^{\prime}\right\|_{\infty} J_{n}^{1 / 2} \\
& \leq 2^{-p} C\left\|\eta^{\prime}\right\|_{\infty} J_{n}^{1 / 2} .
\end{aligned}
$$

Hence it follows from (6.1), (6.2) and this inequality that

$$
\left|\eta_{n}(t) \xi(t)\right| \leq C\left\|\eta^{\prime}\right\|_{\infty} J_{n}^{1 / 2} \sum_{p=0}^{\infty} \frac{1}{2^{p}}=2 C\left\|\eta^{\prime}\right\|_{\infty} J_{n}^{1 / 2}
$$

whenever $t \in(0, \sigma)$. It therefore follows that in the event $\lim _{\inf }{ }_{n \rightarrow \infty} J_{n}=0, \xi=0$ on $(0, \sigma)$, which forces $\xi$ to be identically zero. This takes care of the case where $v$ satisfies condition (I).

Suppose now that $v$ satisfies condition $\left(\mathrm{I}_{\nu}\right)$ for some $\nu>1 / 2$. In this case we define

$$
\begin{aligned}
\left(R_{\nu} g\right)(t) & =\frac{v(t)-v\left(0^{+}\right)}{t^{\nu}} \tilde{\Psi}_{k, b, \lambda}(t) \int_{0}^{t} \tilde{\Psi}_{k, b, \lambda}^{-1}(s) \frac{1}{s^{1-\nu}} \tilde{B}^{-1} g(s) d s \\
& =\left(\left(v-v\left(0^{+}\right)\right) \tilde{\Psi}_{k, b, \lambda} T_{\nu-1} \tilde{\Psi}_{k, b, \lambda}^{-1} \tilde{B}^{-1} g\right)(t)
\end{aligned}
$$

when $t \in(0, d)$. Here,

$$
\left(T_{\nu-1} f\right)(t)=\frac{1}{t^{\nu}} \int_{0}^{t} \frac{1}{s^{1-\nu}} f(s) d s .
$$

Now choose $\sigma \in(0, \delta / 2)$ such that

$$
\left\|\chi_{(0, \sigma)} R_{\nu} \chi_{(0, c)}\right\| \leq 1 / 2,
$$


which is possible because $T_{\nu-1}$ is bounded and $v(\epsilon) \rightarrow v\left(0^{+}\right)$as $\epsilon \rightarrow 0$. For each $n \in \mathbf{N}$, let

$$
f_{n}(t)=\chi_{(0, \sigma)}(t) \frac{v(t)-v\left(0^{+}\right)}{t^{\nu}}\left(R \eta_{n}^{\prime} \tilde{B} \xi\right)(t) .
$$

By (6.2) and the assumption that $\left|v(t)-v\left(0^{+}\right)\right| / t^{\nu}$ is square-integrable near 0 , there is an $M>0$ such that

$$
\left\|f_{n}\right\| \leq M J_{n}^{1 / 2}
$$

for every $n \in \mathbf{N}$.

For any $p \geq 1$ and $t \in(0, \sigma)$, we have

$$
\left(\left(\left(V-V_{b}\right) R\right)^{p} \eta_{n}^{\prime} \tilde{B} \xi\right)(t)=\frac{1}{t^{1-\nu}}\left(R_{\nu}^{p-1} f_{n}\right)(t) .
$$

Therefore

$$
\left(R\left(\left(V-V_{b}\right) R\right)^{p} \eta_{n}^{\prime} \tilde{B} \xi\right)(t)=t^{\nu}\left(\tilde{\Psi}_{k, b, \lambda} T_{\nu-1} \tilde{\Psi}_{k, b, \lambda}^{-1} \tilde{B}^{-1} R_{\nu}^{p-1} f_{n}\right)(t)
$$

whenever $t \in(0, \sigma)$. Combining this with (6.3) and (6.4), we obtain

$$
\left\|\chi_{(0, \sigma)} R\left(\left(V-V_{b}\right) R\right)^{p} \eta_{n}^{\prime} \tilde{B} \xi\right\| \leq \sigma^{\nu} C\left\|T_{\nu-1}\right\|\left(\frac{1}{2^{p-1}}\right) M J_{n}^{1 / 2} .
$$

Taking (6.1) and (6.2) into account, we arrive at the estimate

$$
\left\|\chi_{(0, \sigma)} \eta_{n} \xi\right\| \leq \sigma^{1 / 2} C\left\|\eta^{\prime}\right\|_{\infty} J_{n}^{1 / 2}+\sigma^{\nu} C\left\|T_{\nu-1}\right\| M J_{n}^{1 / 2} \sum_{p=1}^{\infty} \frac{1}{2^{p-1}}
$$

As in the previous case, if $\liminf _{n \rightarrow \infty} J_{n}=0$, then $\chi_{(0, \sigma)} \xi=0$. This completes the proof.

Proposition 6.2. Suppose that $Q$ is a real-valued measurable function on $(0, \infty)$ which has the following property: There exist positive numbers $M, K, \epsilon$ and a such that

$$
a / t^{2-\epsilon} \leq|Q(t)| \leq M / t \text { for a.e. } t \in(K, \infty) .
$$

Furthermore, suppose that $Q$ does not change sign on $(K, \infty)$ and is bounded outside every neighborhood of 0 . Let $A$ be any self-adjoint extension in $\bigoplus_{k=0}^{\ell}\left(L^{2}(0, \infty) \otimes\right.$ $\left.\mathbf{C}^{4}\right)^{[2 k+2]}$ of the symmetric operator $\bigoplus_{k=0}^{\ell}\left(\tilde{D}_{k}-Q\right)^{[2 k+2]}$ on $\bigoplus_{k=0}^{\ell}\left(C_{c}^{\infty}(0, \infty) \otimes\right.$ $\left.\mathbf{C}^{4}\right)^{[2 k+2]}$. Let $E_{A}$ be the spectral measure for $A$. Then

$$
H_{\ell, 0}=P_{\ell, 0} E_{A}\left(-\left|\mu_{0}\right|,\left|\mu_{0}\right|\right) \bigoplus_{k=0}^{\ell}\left(L^{2}(0, \infty) \otimes \mathbf{C}^{4}\right)^{[2 k+2]}
$$

is an infinite dimensional space.

Proof. We claim that it suffices to show that there is an infinte dimensional linear space $\mathcal{Y} \subset\left(C_{c}^{\infty}(0, \infty) \otimes \mathbf{C}^{4}\right)^{[2]} \oplus 0 \oplus \cdots \oplus 0=P_{\ell, 0} \bigoplus_{k=0}^{\ell}\left(C_{c}^{\infty}(0, \infty) \otimes \mathbf{C}^{4}\right)^{[2 k+2]}$ such that $\left\langle\left(A^{2}-\mu_{0}^{2}\right) w, w\right\rangle<0$ for every nonzero $w \in \mathcal{Y}$. Indeed if the dimension of $H_{\ell, 0}$ were finite, then, by elementary linear algebra, there would be an infinite dimensional subspace $\mathcal{Y}_{0} \subset \mathcal{Y}$ such that $\mathcal{Y}_{0} \perp H_{\ell, 0}$. But $\mathcal{Y}_{0}$ is automatically orthogonal to the range of $1-P_{\ell, 0}$. Hence $\mathcal{Y}_{0}$ would be orthogonal to the range of $E_{A}\left(-\left|\mu_{0}\right|,\left|\mu_{0}\right|\right)$. Thus $\left\langle\left(A^{2}-\mu_{0}^{2}\right) w_{0}, w_{0}\right\rangle \geq 0$ for every $w_{0} \in \mathcal{Y}_{0}$, which is a contradiction. 
But to show that such a $\mathcal{Y}$ exists, it suffices to exhibit an infinite dimensional linear subspace $Y \subset C_{c}^{\infty}(0, \infty) \otimes \mathbf{C}^{4}$ such that

$$
\left\langle\left\{\left(\tilde{D}_{0}-Q\right)^{2}-\mu_{0}^{2}\right\} w, w\right\rangle<0
$$

for every $w \neq 0$ in $Y$. For this purpose we first note that, with identity matrices suppressed,

$$
\begin{aligned}
\left(\tilde{D}_{0}-Q\right)^{2}-\mu_{0}^{2} & =D_{0}^{2}+Q^{2}-2 \mu_{0} Q \sigma_{1}-\left(Q D_{0}+D_{0} Q\right) \sigma_{3} \\
& \leq-\frac{d^{2}}{d t^{2}}+P-2 \mu_{0} Q \sigma_{1}-\left(Q D_{0}+D_{0} Q\right) \sigma_{3}
\end{aligned}
$$

where $|P(t)| \leq N / t^{2}$ for some fixed $N>0$ and all $t \in(K, \infty)$. Therefore

$$
\begin{aligned}
& \left\langle\left\{\left(\tilde{D}_{0}-Q\right)^{2}-\mu_{0}^{2}\right\}(\varphi \oplus( \pm \varphi)), \varphi \oplus( \pm \varphi)\right\rangle \\
& \quad \leq\left\langle\left\{-\frac{d^{2}}{d t^{2}}+P-2 \mu_{0} Q \sigma_{1}-\left(Q D_{0}+D_{0} Q\right) \sigma_{3}\right\}(\varphi \oplus( \pm \varphi)), \varphi \oplus( \pm \varphi)\right\rangle \\
& \quad=2\left\langle\left\{-\frac{d^{2}}{d t^{2}}+P \mp 2 \mu_{0} Q\right\} \varphi, \varphi\right\rangle-\left\langle\left(Q D_{0}+D_{0} Q\right)(\varphi \oplus(\mp \varphi)), \varphi \oplus( \pm \varphi)\right\rangle \\
& \quad=2\left\langle\left\{-\frac{d^{2}}{d t^{2}}+P \mp 2 \mu_{0} Q\right\} \varphi, \varphi\right\rangle
\end{aligned}
$$

if $\varphi \in C_{c}^{\infty}(R, \infty) \otimes \mathbf{C}^{2}$. Thus it suffices to consider the case where $\mu_{0} Q>0$ on $(K, \infty)$ and to find an infinite dimensional subspace $Y_{0} \subset C_{c}^{\infty}(R, \infty)$ such that

$$
\left\langle\left\{-\frac{d^{2}}{d t^{2}}+P-2 \mu_{0} Q\right\} u, u\right\rangle<0
$$

for every nonzero $u \in Y_{0}$. For this purpose we use an old trick (see [10, Theorem XIII.6(a)]).

Note that there exist $K_{1}>K$ and $a_{1}>0$ such that

$$
2 \mu_{0} Q(t)-P(t) \geq a_{1} / t^{2-\epsilon} \text { whenever } t>K_{1} .
$$

Let $h \in C_{c}^{\infty}(0, \infty)$ be such that $h=0$ on $(0,1 / 2) \cup(1, \infty)$ and $h$ is not identically zero. For each $S>0$, let $h_{S}(t)=h(t / S)$. Then

$$
-\left\langle\frac{d^{2}}{d t^{2}} h_{S}, h_{S}\right\rangle=\left\langle h_{S}^{\prime}, h_{S}^{\prime}\right\rangle=S^{-2} \int_{0}^{\infty}\left|h^{\prime}(t / S)\right|^{2} d t=S^{-1}\left\|h^{\prime}\right\|^{2}
$$

Since $h_{S}=0$ on $(S, \infty)$, we also have

$$
\left\langle\left(2 \mu_{0} Q-P\right) h_{S}, h_{S}\right\rangle \geq a_{1} S^{-2+\epsilon} \int_{0}^{\infty}|h(t / S)|^{2} d t=a_{1} S^{-1+\epsilon}\|h\|^{2}
$$

when $S / 2>K_{1}$. Therefore, for such an $S$

$$
\left\langle\left\{-\frac{d^{2}}{d t^{2}}+P-2 \mu_{0} Q\right\} h_{S}, h_{S}\right\rangle \leq S^{-1}\left(\left\|h^{\prime}\right\|^{2}-a_{1} S^{\epsilon}\|h\|^{2}\right) .
$$

Since $\|h\|>0$, the above quantity is negative if $S$ is sufficiently large. This implies that there is a sequence $\left\{h_{1}, \ldots, h_{n}, \ldots\right\}$ of $C^{\infty}$-functions with mutually disjoint supports such that (6.6) holds for every $u=h_{n}$. Let $Y_{0}$ be the linear span of $\left\{h_{1}, \ldots, h_{n}, \ldots\right\}$. Because $\left(-d^{2} / d t^{2}+P-2 \mu_{0} Q\right) h_{n}$ and $h_{m}$ also have disjoint supports whenever $n \neq m,(6.6)$ holds for every nonzero $u \in Y_{0}$. This completes the proof. 
Theorem 6.3. Suppose that $\left|v\left(0^{+}\right)\right|>1$ and that $v$ satisfies either the condition (I) or the condition $\left(I_{\nu}\right)$ for some $\nu>1 / 2$. Suppose that $W$ satisfies the condition that

$$
a / r^{2-\epsilon} \leq|W(r)| \leq M / r
$$

when $r>K$, where $a, \epsilon, M$ and $K$ are positive constants. Furthermore, suppose that $W(r)$ does not change sign when $r>K$. Let $A$ be a self-adjoint extension in $L^{2}\left(\mathbf{R}^{3}\right) \otimes \mathbf{C}^{4}$ of the minimal symmetric operator $\alpha \cdot p+\mu_{0} \beta-V-W$. Then there exists an infinite orthonormal set $\left\{\varphi_{1}, \ldots, \varphi_{n}, \ldots\right\}$ consisting of eigenvectors of $A$ corresponding to eigenvalues in $\left(-\left|\mu_{0}\right|,\left|\mu_{0}\right|\right)$ such that $\left|\varphi_{n}(x)\right|^{2} /|x|$ is not integrable on $\mathbf{R}^{3}$ for every $n$.

It is a well-known fact that every $\xi$ in the domain of $\left|\alpha \cdot p+\mu_{0} \beta\right|^{1 / 2}$ has the property that $|\xi(x)|^{2} /|x|$ is integrable on $\mathbf{R}^{3}$. Thus when $V$ and $W$ satisfy the conditions of this theorem, it is impossible to construct any self-adjoint extension $A$ of the minimal operator $\alpha \cdot p+\mu_{0} \beta-V-W$ such that the domain of $A$ is contained in that of $\left|\alpha \cdot p+\mu_{0} \beta\right|^{1 / 2}$. This contrasts sharply with the case where $\left|v\left(0^{+}\right)\right|<1$ [8], [11], [13].

The physical significance of this theorem is that every self-adjoint extension $A$ of $\alpha \cdot p+\mu_{0} \beta-V-W$ possesses an infinite number of eigenstates $\varphi$ corresponding to eigenvalues in $\left(-\left|\mu_{0}\right|,\left|\mu_{0}\right|\right)$ such that the generalized expectation values

$$
\langle V+W\rangle_{\varphi}=\int_{\mathbf{R}^{3}}\langle(V(|x|)+W(|x|)) \varphi(x), \varphi(x)\rangle_{\mathbf{C}^{4}} d m_{3}(x)
$$

and

$$
\langle\alpha \cdot p\rangle_{\varphi}=\int_{\mathbf{R}^{3}}\langle(\alpha \cdot p \varphi)(x), \varphi(x)\rangle_{\mathbf{C}^{4}} d m_{3}(x)
$$

for the potential energy and the kinetic energy fail to be finite. Thus the finite expectation value $\langle A \varphi, \varphi\rangle$ for the total energy is obtained as the result of cancellation between two infinities. In other words, self-adjoint extension somehow assumes the role of renormalization in this situation.

Proof of Theorem 6.3. It is an immediate consequence of Theorem 4.3 that the essential spectrum of $A$ is the same as that of $\alpha \cdot p+\mu_{0} \beta-W$. Since $W$ is bounded and vanishes at infinity, $\left(\alpha \cdot p+\mu_{0} \beta-W-z\right)^{-1}-\left(\alpha \cdot p+\mu_{0} \beta-z\right)^{-1}$ is compact for every $z \in \mathbf{C} \backslash \mathbf{R}$. Hence $\sigma_{\text {ess }}(A)=\sigma_{\text {ess }}\left(\alpha \cdot p+\mu_{0} \beta\right)=\left(-\infty,-\left|\mu_{0}\right|\right] \cup\left[\left|\mu_{0}\right|, \infty\right)$.

Let $\ell \in \mathbf{Z}_{+}$be such that $\left|v\left(0^{+}\right)\right|<\left((2+\ell)^{2}-(1 / 4)\right)^{1 / 2}$. Let $U$ be the unitary operator introduced in Proposition 3.3. Then

$$
\begin{gathered}
U^{[2]}\left(\alpha \cdot p+\mu_{0} \beta-V-W\right) U^{*[2]} \\
=\left\{\bigoplus_{k=0}^{\ell}\left(\tilde{D}_{k}-V-W\right)^{[2 k+2]}\right\} \oplus\left\{\bigoplus_{k=\ell+1}^{\infty}\left(\tilde{D}_{k}-V-W\right)^{[2 k+2]}\right\}
\end{gathered}
$$

By the choice of $\ell$ and Theorem 2.2, the closure $S^{\prime}$ of the second summand above is a self-adjoint operator. Hence there is a self-adjoint extension $S$ of $\bigoplus_{k=0}^{\ell}\left(\tilde{D}_{k}-\right.$ $V-W)^{[2 k+2]}$ such that

$$
U^{[2]} A U^{*[2]}=S \oplus S^{\prime}
$$

In particular $\sigma_{\text {ess }}(S)$ does not intersect $\left(-\left|\mu_{0}\right|,\left|\mu_{0}\right|\right)$. 
By Proposition 6.2 and the assumption on $W, S$ has distinct eigenvalues $\lambda_{1}, \ldots$, $\lambda_{n}, \ldots$ in $\left(-\left|\mu_{0}\right|,\left|\mu_{0}\right|\right)$ which have the following property: For each $n$, there is a unit eigenvector $\xi_{n}$ of $S$ corresponding to the eigenvalue $\lambda_{n}$ such that $P_{\ell, 0} \xi_{n} \neq 0$. Since $v$ satisfies either condition (I) or condition $\left(\mathrm{I}_{\nu}\right)$ for some $\nu>1 / 2$, it follows from Proposition 6.1 that $\left|\xi_{n}(t)\right|^{2} / t$ is not integrable near 0. It we set $\varphi_{n}=U^{*[2]} \xi_{n}$ for each $n$, then $A \varphi_{n}=\lambda_{n} \varphi_{n}$. The non-integrability of $\left|\varphi_{n}(x)\right|^{2} /|x|$ on $\mathbf{R}^{3}$ follows from that of $\left|\xi_{n}(t)\right|^{2} / t$ and the fact that $U^{[2]}$ preserves the multiplication by any radial function. In particular, $\left\langle f \varphi_{n}, \varphi_{n}\right\rangle=\left\langle f \xi_{n}, \xi_{n}\right\rangle$ if $f$ is radial.

To conclude this paper, let us mention that we strongly suspect that Proposition 6.1 and Theorem 6.3 remain valid if the assumption that $v$ satisfies either condition (I) or condition $\left(\mathrm{I}_{\nu}\right)$ is dropped. In other words, the boundedness of $v$ and the condition that $v\left(0^{+}\right)=\lim _{\epsilon \rightarrow 0} v(\epsilon)$ exists should suffice for these propositions. Technically it should be an interesting exercise in analysis to try to prove this.

\section{REFERENCES}

1. C. Bennett and R. Sharpley, Interpolation of operators, Academic Press, Boston, 1988.

2. P. Chernoff, Schrödinger and Dirac operators with singular potentials and hyperbolic equations, Pacific J. Math. 72 (1977), 361-382. MR 89e:46001

3. I. Gohberg and M. Krein, Introduction to the theory of linear nonselfadjoint operators, Amer. Math. Soc. Transl. of Mathematical Monographs 18, Providence, 1969. MR 39:7447

4. H. Kalf, A limit point criterion for separated Dirac operators and a little known result on Riccati's equation, Math. Z. 129 (1972), 75-82. MR 47:3745

5. H. Kalf, U.-W. Schmincke, J. Walter and R. Wüst, On the spectral theory of Schrödinger and Dirac operators with strongly singular potentials, Lecture Notes in Mathematics 448, Springer-Verlag, Berlin, 1975. MR 53:1031

6. T. Kato, Perturbation theory for linear operators, Springer-Verlag, New York, 1976. MR 53:11389

7. M. Naimark, Linear differential operators, Part II: Linear differential operators in Hilbert space, Ungar, New York, 1968. MR 41:7485

8. G. Nenciu, Self-adjointness and invariance of the essential spectrum for Dirac operators defined as quadratic forms, Commun. Math. Phys. 48 (1976), 235-247. MR 54:9459

9. M. Rose, Relativistic electron theory, Wiley, New York, 1961.

10. M. Reed and B. Simon, Methods of modern mathematical physics, IV: Analysis of operators, Academic Press, New York, 1978. MR 58:12429c

11. U.-W. Schmincke, Distinguished self-adjoint extensions of Dirac operators, Math. Z. 129 (1972), 335-349. MR 48:4792

12. E. Titchmarsh, On the nature of the spectrum in problems of relativistic quantum mechanics, Quart. J. Math. Oxford 12 (1961), 227-240. MR 26:5211

13. R. Wüst, Distinguished self-adjoint extensions of Dirac operators constructed by means of cut-off potentials, Math. Z. 141 (1975), 93-98. MR 51:1486

Department of Mathematics, State University of New York at Buffalo, Buffalo, New York 14214

E-mail address: jxia@acsu.buffalo.edu 